Sharif University of Technology
Scientia Iranica
Transactions E: Industrial Engineering
http://scientiairanica.sharif.edu
IRAN ICA

\title{
Developing a mathematical model for staff routing and scheduling in home health care industries: Genetic algorithm-based solution scheme
}

\author{
Z. Entezari a and M. Mahootchi* \\ Department of Industrial Engineering \& Management Systems, Amirkabir University of Technology (Tehran Polytechnic), Tehran, \\ Iran.
}

Received 27 July 2019; received in revised form 13 November 2019; accepted 3 March 2020

\section{KEYWORDS \\ Home health care; \\ Routing and \\ scheduling; \\ Double services; \\ Temporal \\ interdependencies; \\ Genetic algorithm.}

\begin{abstract}
Efficient management of providing home health care services requires many considerations. In this paper, a mathematical model for the daily staff routing and service scheduling is developed for home health care companies. In this model, both economic factors and qualitative service-oriented performance measures are simultaneously optimized. To make the model more realistic, many real situations such as considering different qualifications and diverse vehicles for staff members, different requirements and predetermined preferences for patients, possible temporal interdependencies between services, and Continuity of Care $(\mathrm{CoC})$ are taken into account. We also added some important constraints related to blood sampling requirements, which make our proposed model more complex. The proposed model is a Mixed-Integer Linear Programming (MILP) model that belongs to an NP-hard class of optimization problems. To solve such a complex mathematical model, a Genetic Algorithm (GA) is proposed to find near-optimal solutions. We use some randomly generated test instances with different sizes to evaluate the performance of the GA. Finally, it is demonstrated how the proposed solution scheme can end up with proper scheduling and routing policies compared to those obtained through exact methods.
\end{abstract}

(C) 2021 Sharif University of Technology. All rights reserved.

\section{Introduction}

How to provide services from a certain depot to the demand points has always been one of the main topics in transportation systems. The demand for providing services at home has remarkably grown in recent years. Furthermore, there are many changes in the socio-cultural and economic lifestyles of people in developed and developing countries. Therefore, the

\footnotetext{
*. Corresponding author. Tel.: +98 2164545387 E-mail addresses: entezari.z@aut.ac.ir (Z. Entezari); mmahootchi@aut.ac.ir (M. Mahootchi)
}

traditional pattern of delivering health care services should inevitably change to a new and enhanced home health care pattern. Home health care, as an important issue in the supply chain, can be viewed as a logistics network with different entities: a home health care company, where the process of delivering services is initiated, and the demanding points, where the caring services are delivered to the patients. The main reasons for the increasing demand for home health care services would be summarized as follows [1]:

1. The limited capacity of hospitals and remarkable growth in world populations which leads to high expenses for hospitalization;

2. An increase in life expectancy due to enhancement 


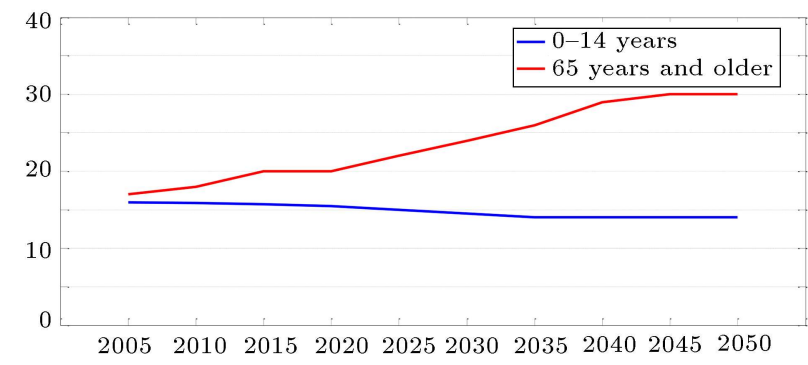

Figure 1. The proportion of the population in the European Union (27 countries).

of nutrition plans and improving lifestyles, which leads to more elderly people in society;

3. Changing the preferences of people to receive services in their homes instead of going to hospitals and walk-in clinics.

Among the aforementioned items, the increasing size of the elderly population would be the most prominent factor, which could make home health care quite beneficial and attractive for business practitioners and academic researchers. Figure 1 demonstrates an increasing trend in older people in many European countries from 2005, which is expected to continue to 2050 [1].

Today, many home health care companies should provide a wide variety of services to patients; therefore, they have to employ staff members with various qualifications and skills (e.g., a person having permission to prescribe medications or having enough ability to do bathing for special elderly patients, professional nurses, specific physicians, chiropractors, and massagers). Furthermore, whereas staff members are manually scheduled, the time schedules are not usually reliable which means that the required services and proper assignment of human resources (i.e., staff members) could not be suitably accomplished $[2,3]$. The home health care companies should also consider the level of qualification and time window (i.e., considering available periods for patients and staff members) for each service. This means that the required services for a patient should be provided by eligible staff members with consideration of the desired time windows for both respective patients and staff members. Some interdependent services that should be performed in serial or parallel orders could exist.

Moreover, one can choose the staff member based on the genders or mother tongues, which could be an important issue for some countries with more emigrants such as Canada. In this case, the respective patient chooses the features of the staff members such as her/his tongue or gender, and the manager of the home health care center should specify which staff members would handle the related service in the preprocessing phase of the model construction. In other words, more than a single staff member would be eligible to perform the service. It can also be assumed that patients have already registered and experienced the respective requirements in the past days or have some information about the staff members via their relatives or friends. Considering all these aforementioned issues, which would be called the patients' preferences, is recognized as Continuity of Care $(\mathrm{CoC})$ in the home health care literature. This is a challenging issue in health care services, in which the staff member who is in favor of the patient should provide some required services. By adding the performance measure of $\mathrm{CoC}$, both patients and staff members would have more friendly relations. This feature is usually incorporated into the optimization model for long-term perspectives. However, addressing it in a daily-based model would be problematic and questionable. The main reasons for considering $\mathrm{CoC}$ in the daily scheduling of home health care problems, as we mentioned, would be the patient experiences or the information obtained via their relatives or friends for a staff member. In fact, some patients are quite familiar with the staff members who provide the required services at the home health center. Therefore, $\mathrm{CoC}$ could be taken into consideration for the daily planning horizon in which this advantage is given to patients to nominate favorite staff members for their requirements.

Few studies have considered all the above limitations of the objectives mentioned in the optimization mode [4-6]. Specifically, considering interdependent services (the necessity of performing two services simultaneously or in a specific order) becomes a hot topic in providing home health care services (e.g., papers Kergosien et al. [5], Rasmussen et al. [6], and Mankowska et al. [7]). The staff working plans are usually manipulated based on trial and error to be matched with required interdependency between services; as a result, the quality of the plan could decrease significantly. Moreover, in a few papers, the use of $\mathrm{CoC}$ for home healthcare issues is considered a basic performance measure [8-10]. Here, it should be mentioned that the Home Healthcare Routing and Scheduling Problem (HHCRSP) in this situation could be very distinct from the well-known multiple traveling salesman problem with time windows. In our problem, the patients requirements must be compatible with the qualifications of the staff members. Furthermore, a patient would need several dependent/independent requirements and there is a possibility that a staff could visit a patient several times. All these changes make this problem specific, which cannot be tackled with developed solution schemes used for solving travelling salesman problem.

In this paper, MILP model is proposed for the HHCRSP. The goals are to determine the daily route of each staff member and to schedule the service requirements of the patients. Moreover, the compat- 
ibility between staff members' qualifications and the requirements, which should be occurred at a certain time horizon, are taken into consideration in our proposed model. In our proposed formulation, a single staff member must perform each required service by one patient (i.e., single service). In addition, interdependent services are incorporated into the model in two different ways. The first one includes the services requiring two staff members at the same time, called simultaneous services (e.g., bathing and lifting a disabled patient). The second includes all services that should be executed within a predetermined time distance with a specific priority (e.g., giving medication to a patient at a certain time before/after a meal with a one-hour time distance between them).

In general, we develop a mathematical formulation considering heterogeneous staff members, possible interdependencies between services, and various travel times depending on vehicle speeds. The model also considers the specific limitations pertinent to getting the blood samples. To the best of our knowledge, this is the first attempt to consider all these limitations in a mathematical model. A Genetic Algorithm (GA) is also developed to solve this complex problem. The remainder of the paper is organized as follows: Section 2 presents a literature review of the latest research related to HHCRSP. Section 3 is devoted to explain all respective assumptions, the parameters, and the decision variables and to present the mathematical formulation. Due to the high complexity of the model, a GA is extended and explained in detail in Section 4. Section 5 is dedicated to computational results by which the efficiency of the model and the proposed solution scheme are investigated. Finally, conclusions are made and some possible suggestions for further research are recommended in Section 6.

\section{Literature review}

A survey of the literature shows that the research on the HHCRSP has relatively attracted less attention compared to various Vehicle Routing Problems (VRP) [11-14] categorized home care logistics management problems as operational decisions including transportation and staff logistics functions. Staff routing is one of the decisions included in "transportation management". The routing problem that considers the time window of each service, the frequency, the number of repetitions, and the duration of the service leads to routing and scheduling problems. The optimal routing and scheduling decisions could be very influential in reducing costs and satisfying the patients and that is why many practitioners in-home health care industries mainly focus on finding the optimal/near-optimal solutions for these two decisions. For further information, the readers could refer to Gutiérrez and Vidal [14].
Moreover, Fikar and Hirsch [15] conducted a thorough and detailed review of the home health care routing and scheduling papers and classified them into two groups: single- and multi-period. All research studies in this review paper are categorized in terms of different characteristics (e.g., temporal precedence, visiting patterns, multi-objective, and dynamic or stochastic settings). They also discussed different objectives, constraints, and the respective solution methods used in home health care problems.

The followings present some important papers addressing the HHCRSP. Begur et al. [16] were the first researchers who presented a decision support system for tour and roster planning. The goals were to minimize the total travel time and balance the workload of heterogeneous staff members. The heuristic approach proposed for solving the problem was based on both savings heuristics and nearest neighbor approaches in order to re-optimize each route. Cheng and Rich [17] presented a MILP model for the problem of determining an optimal schedule with the aim of minimizing the total cost of overtime (for full-time staff) and parttime work (for part-time staff) and the total distance traveled by staff. In this paper, a two-phase heuristic approach was also proposed to tackle such a problem. They found an initial solution using a randomized greedy algorithm in the first phase and improved it using a local search method in the second phase. Bertels and Fahle [18] proposed a combination of linear programming and constraint programming to find initial solutions for an assignment and routing problem. These solutions were improved by two metaheuristic approaches: tabu search and simulated annealing. The objectives were to minimize the total travel time and to maximize the satisfaction of both patients and staff members. Eveborn et al. [19] described an operational system called LAPSCARE for both patients allocation to staff members and visit schedules. They formulated the problem using a set-partitioning model to assign the patients to the routes of the available staff. A repeated matching heuristic was presented to solve the problem. Their main goals were to reduce the total travel time and to increase patients satisfaction. Bräysy [20] investigated a real-world case in Finland for home care services and used a commercial solver to determine the routing of staff members in different scenarios. Akjiratikarl et al. [21] modeled a home health care problem in the UK with the aim of minimizing the traveling distance. They presented a solution scheme based on particle swarm optimization to find near-optimal scheduling plans for visiting patients. Furthermore, a mathematical programming model was presented by Bredström and Rönnqvist [4] for routing and scheduling of vehicles. Kergosien et al. [5] also extended the multiple traveling salesman problems to be matched to the home health care case in which 
the number of nodes was exactly equal to the number of required services. Trautsamwieser and Hirsch [22] presented a mathematical model for nurses scheduling using real data in Austria. Elbenani et al. [9] proposed a mathematical model for the HHCRSP and used the scatter search approach to solve such a problem. In this paper, some specific medical restrictions were considered about the requirements of returning all blood samples to the respective laboratory within a certain time interval. Furthermore, each nurse and each patient should be assigned to one section according to their home address. The objectives were to minimize the total travel time, the daily cost for the different nurses, a penalty cost for violation of $\mathrm{CoC}$, and a penalty cost for each patient in a section visited by a nurse from different sections. Rasmussen et al. [6] formulated the home health care problem using the set-partitioning model and developed a solution method based on the branch and price approach to find the optimal routing and scheduling solution. Their model could bring the temporal dependences into different types of optimization models. The goals were to minimize the total traveling costs and the uncovered visits as well as to maximize the staff-visit preferences. Mankowska et al. [7] presented a comprehensive mathematical formulation for finding the optimal daily planning of staff members. The objectives were to minimize the total travelling distance and total tardiness and maximal tardiness that occurred in providing services. They solved the model using an effective heuristic method. Hiermann et al. [23] developed a general framework in the home health care context for a realworld multimodal scheduling problem. In this paper, a two-stage approach was also proposed to solve such a problem by considering staff and customer satisfaction. They used stochastic procedures or constraint programming techniques to generate initial solutions in the first stage and improved them in the second stage by one of four metaheuristics (memetic algorithm, variable neighborhood search, scatter search, and a simulated annealing hyper-heuristic).

Duque et al. [24] studied a home care planning problem faced by Landelijke Thuiszorg, a nonprofit organization that delivers home care services in several Belgian regions. Each region had its own region manager, who should schedule proper actions for available caregivers. This problem was formulated based on a set partitioning problem that aims to maximize the service level and minimize the total distance traveled by the caregivers. Afterward, a two-stage solution strategy had been designed to efficiently solve the optimization model with multiple objectives in a hierarchical order which was developed for the planning problem. Furthermore, Redjem and Marcon [25] addressed the challenges of routing and scheduling problem under coordination and precedence constraints in which patients could need multiple staff members for their requirements. In this paper, the visits were performed simultaneously and probably in a predetermined order. To solve the staff members routing problem, they developed a new heuristic approach where its complexity was not sensitive to the temporal dependencies rate. In addition, Frifita et al. [26] provided a general variable neighborhood search to solve the HHCRSP with synchronization and time windows constraints. In this paper, each patient required multiple visits and some visits needed more than a single staff member. Accordingly, the staff members should have the same starting and finishing times (synchronization of the arrival of staff members). The main objective of the model was to minimize the total travel times. Decerle et al. [27] proposed a mixedinteger programming model for HHCRSP with hard and soft patients time window and synchronization constraints. The objectives were to minimize the total travel time and the penalties obtained through violating the patients time window and the synchronized visits. They presented a memetic algorithm to solve the developed model. Moreover, Liu et al. [28] developed a route-based mathematical programming model for the caregiver routing and scheduling problem with stochastic travel and service times. They used a chance constraint to cope with the uncertainty of the travel and service times. The goals were to minimize the expected total operational cost of selected routes and the penalty value for non-visiting customers.

Also, a dynamic approach was proposed by Demirbilek et al. [29]. Moreover, performing a certain visit and transporting medical facilities and medicine between the health care institutions and the patients homes are discussed by Regis-Hernández et al. [30] who solved the dimensioning problem for innovative HHC services with devices and disposable materials, as a joint dimensioning of human and material resources.

Finally, we focus on reviewing the three important aspects of HHCRSP: (1) performance measures or objectives, (2) decisions and constraints, and (3) solution schemes. For this purpose, we add three tables in which our research study is compared to the most relevant papers in the field in terms of the objective functions, the constraints, and the solution schemes. Table 1 demonstrates most objectives or performance measures handled in the reviewed papers. It should be noted that each group comprises an extensive class of possible performance measures or objective functions. For example, time measures could encompass travel and waiting times, and cost measures could consist of travel, assignment, and scheduling costs. Each of the objective functions used in a paper is shown with a symbol $(\sqrt{ })$ in the pertinent cell. As illustrated in this table, the authors have mainly focused on time, cost, and preferences. 
Table 1. Performance measures and objectives considered in the literature.

\begin{tabular}{|c|c|c|c|c|c|c|c|}
\hline \multirow[b]{2}{*}{ Paper } & \multicolumn{7}{|c|}{ Performance measures } \\
\hline & $\begin{array}{c}\text { Cost } \\
\text { measures }\end{array}$ & $\begin{array}{c}\text { Time } \\
\text { measures }\end{array}$ & $\begin{array}{l}\text { Distance } \\
\text { travelled }\end{array}$ & $\begin{array}{c}\text { Patient } \\
\text { preferences }\end{array}$ & $\begin{array}{c}\text { Staff } \\
\text { preferences }\end{array}$ & $\begin{array}{l}\text { Workload } \\
\text { balancing }\end{array}$ & $\begin{array}{c}\text { Uncovered } \\
\text { visits }\end{array}$ \\
\hline Begur et al. [16] & - & $\sqrt{ }$ & - & - & - & $\sqrt{ }$ & - \\
\hline Cheng and Rich [17] & $\sqrt{ }$ & - & $\sqrt{ }$ & - & - & - & - \\
\hline Bertels and Fahle [18] & - & - & $\sqrt{ }$ & $\sqrt{ }$ & $\sqrt{ }$ & - & - \\
\hline Eveborn et al. [19] & $\sqrt{ }$ & - & $\sqrt{ }$ & $\sqrt{ }$ & $\sqrt{ }$ & - & - \\
\hline Bräysy [20] & - & - & $\sqrt{ }$ & - & $\sqrt{ }$ & - & - \\
\hline Akjiratikarl et al. [21] & - & - & $\sqrt{ }$ & - & - & - & - \\
\hline Bredström and Rönnqvist [4] & - & $\sqrt{ }$ & $\sqrt{ }$ & $\sqrt{ }$ & $\sqrt{ }$ & $\sqrt{ }$ & - \\
\hline Kergosien et al. [5] & - & - & $\sqrt{ }$ & - & - & - & - \\
\hline Redjem and Marcon [25] & - & $\sqrt{ }$ & - & - & - & - & - \\
\hline Trautsamwieser and Hirsch [22] & - & - & $\sqrt{ }$ & $\sqrt{ }$ & $\sqrt{ }$ & - & - \\
\hline Elbenani et al. [9] & $\sqrt{ }$ & $\sqrt{ }$ & - & - & - & $\sqrt{ }$ & - \\
\hline Rasmussen et al. [6] & $\sqrt{ }$ & - & - & - & $\sqrt{ }$ & - & $\sqrt{ }$ \\
\hline Liu et al. $[36]$ & $\sqrt{ }$ & - & - & - & - & - & - \\
\hline Mankowska et al. [7] & - & - & $\sqrt{ }$ & $\sqrt{ }$ & - & - & - \\
\hline Decerle et al. [27] & $\sqrt{ }$ & $\sqrt{ }$ & - & - & - & - & - \\
\hline Liu et al. [28] & $\sqrt{ }$ & - & - & - & - & - & - \\
\hline This paper & - & $\sqrt{ }$ & $\sqrt{ }$ & $\sqrt{ }$ & $\sqrt{ }$ & - & - \\
\hline
\end{tabular}

Table 2. Constraints and decisions considered in the literature.

\begin{tabular}{|c|c|c|c|c|c|c|}
\hline \multirow{3}{*}{ Paper } & \multicolumn{6}{|c|}{ Constraints and decisions } \\
\hline & \multirow{2}{*}{$\begin{array}{c}\text { Time } \\
\text { windows }\end{array}$} & \multirow{2}{*}{ Compatibility $^{\mathbf{a}}$} & \multirow{2}{*}{$\begin{array}{c}\text { Staff/patient } \\
\text { preference }\end{array}$} & \multicolumn{2}{|c|}{ Interdependency } & \multirow{2}{*}{$\begin{array}{l}\text { Blood sampling } \\
\text { requirements }\end{array}$} \\
\hline & & & & Simultaneity & Precedence & \\
\hline Begur et al. [16] & $\sqrt{ }$ & $\sqrt{ }$ & - & - & - & - \\
\hline Cheng and Rich [17] & - & $\sqrt{ }$ & - & - & - & - \\
\hline Bertels and Fahle [18] & - & $\sqrt{ }$ & $\sqrt{ }$ & - & - & - \\
\hline Eveborn et al. [19] & $\sqrt{ }$ & $\sqrt{ }$ & $\sqrt{ }$ & $\sqrt{ }$ & - & - \\
\hline Bräysy [20] & - & $\sqrt{ }$ & $\sqrt{ }$ & - & - & - \\
\hline Akjiratikarl et al. [21] & $\sqrt{ }$ & - & - & - & - & - \\
\hline Bredström and Rönnqvist [4] & $\sqrt{ }$ & - & - & $\sqrt{ }$ & $\sqrt{ }$ & - \\
\hline Kergosien et al. [5] & $\sqrt{ }$ & $\sqrt{ }$ & - & $\sqrt{ }$ & - & - \\
\hline Redjem and Marcon [25] & $\sqrt{ }$ & - & - & - & - & - \\
\hline Trautsamwieser and Hirsch [22] & $\sqrt{ }$ & $\sqrt{ }$ & $\sqrt{ }$ & - & - & - \\
\hline Elbenani et al. [9] & $\sqrt{ }$ & - & $\sqrt{ }$ & - & - & $\sqrt{ }$ \\
\hline Rasmussen et al. [6] & $\sqrt{ }$ & $\sqrt{ }$ & $\sqrt{ }$ & $\sqrt{ }$ & $\sqrt{ }$ & - \\
\hline Liu et al. [36] & $\sqrt{ }$ & - & - & $\sqrt{ }$ & $\sqrt{ }$ & - \\
\hline Mankowska et al. [7] & $\sqrt{ }$ & $\sqrt{ }$ & $\sqrt{ }$ & $\sqrt{ }$ & $\sqrt{ }$ & \\
\hline Decerle et al. [27] & $\sqrt{ }$ & - & - & $\sqrt{ }$ & - & - \\
\hline Liu et al. [28] & $\sqrt{ }$ & - & - & - & - & - \\
\hline This paper & $\sqrt{ }$ & $\sqrt{ }$ & $\sqrt{ }$ & $\sqrt{ }$ & $\sqrt{ }$ & $\sqrt{ }$ \\
\hline
\end{tabular}

a Compatibility between staff members' qualifications and the requirements.

Afterward, some of the most common decisions and constraints considered in the papers are presented in Table 2. As it is observed, providing high-quality services has received more attention by satisfying some constraints such as the compatibility between staff members' qualifications and the requirements, time windows, and patient/staff preferences. However, some real-life constraints (e.g., blood sampling requirements) have received relatively less attention.

Table 3 demonstrates the solution approaches considered in the reviewed papers, which are categorized into three general groups: exact methods, metaheuristics, and hybrid methods, i.e., a combination of two previous methods. According to Table 3, the group of metaheuristics is the most popular category in reallife applications.

Finally, the important general information of some respective research studies is summarized in Table 4. Homogenous staff members mean that all available caregivers have the same skills. That is, there 
Table 3. Solution approaches considered in the literature.

\begin{tabular}{|c|c|c|c|}
\hline \multirow[b]{2}{*}{ Paper } & \multicolumn{3}{|c|}{ Solution approaches } \\
\hline & Exact methods & $\begin{array}{c}\text { (Metaheuristic } \\
\text { methods }\end{array}$ & $\begin{array}{c}\text { Hybrid } \\
\text { methods }\end{array}$ \\
\hline Begur et al. [16] & - & $\sqrt{ }$ & - \\
\hline Cheng and Rich [17] & - & - & $\sqrt{ }$ \\
\hline Bertels and Fahle [18] & - & - & $\sqrt{ }$ \\
\hline Eveborn et al. [19] & - & - & $\sqrt{ }$ \\
\hline Bräysy [20] & - & - & - \\
\hline Akjiratikarl et al. [21] & - & $\sqrt{ }$ & - \\
\hline Bredström and Rönnqvist [4] & - & - & $\sqrt{ }$ \\
\hline Kergosien et al. [5] & - & - & $\sqrt{ }$ \\
\hline Redjem and Marcon [25] & $\sqrt{ }$ & - & - \\
\hline Trautsamwieser and Hirsch [22] & - & $\sqrt{ }$ & - \\
\hline Elbenani et al. [9] & - & $\sqrt{ }$ & - \\
\hline Rasmussen et al. [6] & $\sqrt{ }$ & - & - \\
\hline Liu et al. [36] & - & $\sqrt{ }$ & - \\
\hline Mankowska et al. [7] & - & $\sqrt{ }$ & - \\
\hline Decerle et al. [27] & - & $\sqrt{ }$ & - \\
\hline Liu et al. [28] & $\sqrt{ }$ & - & - \\
\hline This paper & - & $\sqrt{ }$ & - \\
\hline
\end{tabular}

Table 4. Overall comparison of the papers published in the area of Home Healthcare Routing and Scheduling Problem (HHCRSP).

\begin{tabular}{|c|c|c|c|c|c|c|c|}
\hline \multirow[b]{2}{*}{ Paper } & \multicolumn{2}{|c|}{ Staff } & \multirow[b]{2}{*}{ Interdependency } & \multicolumn{2}{|c|}{ Time windows for } & \multicolumn{2}{|c|}{ Performance measures } \\
\hline & Hom. ${ }^{a}$ & Het. $^{b}$ & & Staff & Patient & $\begin{array}{c}\text { Cost } \\
\text { measures }\end{array}$ & $\begin{array}{c}\text { Service quality } \\
\text { measures }\end{array}$ \\
\hline Begur et al. [16] & - & $\sqrt{ }$ & - & - & - & $\sqrt{ }$ & - \\
\hline Cheng and Rich [17] & - & $\sqrt{ }$ & - & - & $\sqrt{ }$ & $\sqrt{ }$ & - \\
\hline Bertels and Fahle [18] & - & $\sqrt{ }$ & - & $\sqrt{ }$ & $\sqrt{ }$ & $\sqrt{ }$ & $\sqrt{ }$ \\
\hline Eveborn et al. [19] & - & $\sqrt{ }$ & $\sqrt{ }$ & - & $\sqrt{ }$ & $\sqrt{ }$ & $\sqrt{ }$ \\
\hline Bräysy [20] & - & $\sqrt{ }$ & - & - & - & $\sqrt{ }$ & - \\
\hline Akjiratikarl et al. [21] & $\sqrt{ }$ & - & - & $\sqrt{ }$ & $\sqrt{ }$ & $\sqrt{ }$ & - \\
\hline Bredström and Rönnqvist [4] & $\sqrt{ }$ & - & $\sqrt{ }$ & $\sqrt{ }$ & $\sqrt{ }$ & $\sqrt{ }$ & $\sqrt{ }$ \\
\hline Kergosien et al. [5] & - & $\sqrt{ }$ & $\sqrt{ }$ & $\sqrt{ }$ & $\sqrt{ }$ & $\sqrt{ }$ & - \\
\hline Trautsamwieser and Hirsch [22] & - & $\sqrt{ }$ & - & $\sqrt{ }$ & $\sqrt{ }$ & $\sqrt{ }$ & $\sqrt{ }$ \\
\hline Elbenani et al. [9] & $\sqrt{ }$ & - & - & $\sqrt{ }$ & $\sqrt{ }$ & $\sqrt{ }$ & $\sqrt{ }$ \\
\hline Rasmussen et al. [6] & $\sqrt{ }$ & - & $\sqrt{ }$ & $\sqrt{ }$ & $\sqrt{ }$ & - & $\sqrt{ }$ \\
\hline Mankowska et al. [7] & - & $\sqrt{ }$ & $\sqrt{ }$ & - & $\sqrt{ }$ & $\sqrt{ }$ & $\sqrt{ }$ \\
\hline Decerle et al. [27] & - & $\sqrt{ }$ & - & - & $\sqrt{ }$ & $\sqrt{ }$ & $\sqrt{ }$ \\
\hline Liu et al. [28] & - & $\sqrt{ }$ & - & - & $\sqrt{ }$ & $\sqrt{ }$ & $\sqrt{ }$ \\
\hline This paper & - & $\sqrt{ }$ & $\sqrt{ }$ & $\sqrt{ }$ & $\sqrt{ }$ & $\sqrt{ }$ & $\sqrt{ }$ \\
\hline
\end{tabular}

a Hom.: Homogeneous; ${ }^{b}$ Het.: Heterogeneous.

are no differences between the staff who perform the requested services. While heterogeneous staff members mean that there are caregivers with different capabilities and some of them don't have permission to do some requested services. As it is observed in Table 1, the majority of the papers related to the HHCRSP assume that the services required by a patient are independent.
However, if temporal interdependencies are properly incorporated into the home health care model, it will be more realistic, therefore appropriate-decisions can be made. In addition, the issue of considering interdependent services in family health care received a great deal of attention by Eveborn et al. [19], Bredström and Rönnqvist [4], Kergosien et al. [5], Rasmussen 
et al. [6], and Mankowska et al. [7]. Furthermore, most of the aforementioned research studies consider both the cost of providing services and the quality of services $[4,6,7,9,18,19,22,27]$. The total distance/time traveled by staff members is usually a measure for the operational costs. However, the patients preferences (i.e., meeting the patient requirements within predetermined time windows and having $\mathrm{CoC}$ ) and the level of satisfying staff members (i.e., visiting patients in the desired time window by staff members) are some important quality measures. Only a few papers took the desired time windows for patients/staff members and temporal interdependencies among services into account [4-6].

We also use a GA-based algorithm to solve our proposed routing and scheduling problem. GA is one of the common methods to solve the VRP. The following papers are some of the well-known samples in which GAs are used in routing problems. Baker and Ayechew [31] investigated the application of a GA for the VRP with a time window and demonstrated that this algorithm could compete with tabu search and simulated annealing. Liu et al. [32] studied the fleet size and mix VRP and presented a GA for solving the problem with a single depot and heterogeneous fleet. Ghoseiri and Ghannadpour [33] presented a biobjective VRP with a soft time window in which goal programming method and GA were considered to be a solution. Tasan and Gen [34] presented a geneticbased method to solve the Vehicle Routing Problem with Simultaneous Pickup and Delivery (VRPSPD). Wang and Chen [35] proposed a model for VRPSPD with a hard time window. They used GA to cope with the model with a single depot. Also, Liu et al. [36] presented two mixed-integer programming models for VRPSPD with a hard time window. GA and tabu search algorithms were two solution methods they used to cope with their proposed models of home healthcare problems. In another paper, Pankratz [37] proposed a group GA that uses a time window to solve VRPSPD.

\subsection{Contributions}

In this paper, we develop a comprehensive MILP model for the HHCRSP, taking into account the time window of staff and patients, and increasing the time interdependency between services (i.e., simultaneous services or services with predefined precedence). The model considers different qualifications for staff members and different requirements for patients who could have their own preferences (e.g., CoC). However, in our paper, this is assumed that most patients are quite familiar with the staff members providing the required services in the home health care center. In addition, the travel times are computed based on the mode of transportation. The model generates routing and scheduling solutions in which all services should be performed. In fact, although performing some required services could be delayed, all patients must be ultimately visited by at least one qualified staff member within contract working time or overtime periods. The model also considers the break-time (mealtime or rest time) for all staff members. Some other medical restrictions in which all blood samples should be returned to the designed laboratory in a specific time interval are also imposed into the model. Indeed, we attempt to provide an integrated model for the HHCRSP involving the essential characteristics that occur in reality but are often neglected. There are some papers that have considered some of the reallife constraints; however, to the best of our knowledge, we have not seen a comprehensive paper including all these constraints together. In other words, we believe that this research paper is the first attempt to bring all these real-life constraints to a single optimization problem. It should also be noted that the developed model with such constraints belongs to the category of NP-hard problems. Therefore, we extend a GA-based solution scheme to solve the proposed optimization model for real-life problems within a reasonable time, suggested by Liu et al. [28]. The followings are the main important advantages of our research study compared with other studies:

- We considered one single node for each patient with one required service. For example, in the case of two requirements for a patient, we considered two nodes called virtual nodes as they are not limited to a patient and include services as well. These changes are performed in the preprocessing phase which makes the model more manageable;

- There is no limitation in our proposed model to apply it in daily, weekly, or even monthly home health care routing and scheduling problems. In a weekly or monthly home health care problem, it is assumed that a patient could be visited multiple times within a week or month. In other words, multiple nodes could be considered in the network for a patient having multiple visits in a week or month. The relation between these nodes is handled based on the type of interdependency and the time windows determined by patients and staff members for each visit. For instance, in the preprocessing phase of making the optimization model, in the case of two visits in a week with a two-day time interval, the minimum and maximum time distance between these two nodes should be set to two and seven days, respectively;

- CoC could be taken into consideration for the daily planning horizon in which this advantage is given to patients to nominate favorite staff members for their requirements. In daily scheduling, we assume that patients have already registered and experienced 
the respective requirements in past days or have some information about the staff members via their relatives or friends. In fact, some patients are quite familiar with the staff members providing the required services in the home health care center;

- There is also no restriction to consider different blood, break, and depot centers for each staff member. The proposed optimization model could be simply customized for the new problems of multiple nodes in blood, interruption, and storage centers. Each of these nodes has its own time window and time duration exactly the same as service nodes that should be specifed by the manager before making the optimization model.

\section{Modeling the problem}

In this section, we first start with the problem definition and then continue with considered assumptions for making the model. All needed notations and the mathematical formulation of the developed optimization model are then presented.

\subsection{Problem definition}

The HHCRSP is defined by the completed graph $(N, E)$ where $N$ is composed of a central depot (i.e., home health care company) and patients, and $E$ is the set of edges between two nodes. In this network, staff members are dispatched from the central depot to the patients homes to provide service requirements. Without loss of generality, we can consider multiple locations as central depots without any amendments in the optimization model in terms of the constraints and the objective function. In our proposed formulation, each node represents one required service by one patient, that is, there is only a single service in each node, which must be performed by a single staff.

In fact, the goal of the HHCRSP is to determine the staff routes and schedule the requirements of the logistics network in a certain time horizon on a daily basis, such that the virtual penalties and real costs are minimized. If the total tardiness of providing services to patients (virtual cost) is minimized, it can indicate that good responsiveness to patients is a vital issue in the HHCRSP. Travel time (real cost) should be decreased based on distance traveled by staff and the speed of his/her vehicle. In general, there are two types of measures for providing home health care services: (1) travel time-oriented and (2) service quality-oriented. In this planning problem, each service should be performed in a given time window. The staff members are considered heterogeneous, that is, each staff is qualified for certain services. In addition, each patient has particular requirements. Generally, patients are divided into two groups with respect to their service requirements: patients requiring single services (e.g., single visit), and those requiring interdependent services (e.g., bathing and giving medication to a patient before/after a meal with a predefined time distance between them).

\subsubsection{Assumptions}

- The vehicles could have different velocities, that is, a different type of vehicles could be taken into consideration (i.e., fleets are heterogeneous);

- The model is developed for a single depot, that is, all staff members should start their travels for providing required services from a specific location;

- There are bidirectional connections between all possible pairwise nodes (i.e., each staff member could travel from any node to other nodes);

- Four virtual nodes are defined: $0, n+1$, blood, break. They correspond to the initial and the end nodes of the route, the destination node related to returning the blood samples, and the destination node related to breaking (mealtime or rest time), respectively;

- Each node represents one service, which must be assigned to only one staff member. In other words, in the case of more than one service for a patient, it could virtually become different from multiple nodes with zero distances;

- The optimization model is constructed for routing and scheduling of home health care center in a specified area where all staff members live near the center of providing services.

\subsection{Preprocessing phase}

In this paper, the aim is to consider a more general state of the routing and scheduling problem for home health care services. Generally, a patient could require several services of the same type, or different types, which can be interdependent, or independent from each other. To incorporate all these situations into a mathematical model, we generate a matrix showing the service requirements of all patients such that there is only one identity element in each row of the matrix. This means that there could be different rows pertinent to one patient; however, in each row, only one service can be assigned. In other words, from a logistics network point of view, each row of the matrix represents a single node in the network, indicating a pair of a patient and a service requirement.

Suppose that we have a logistics network consisting of $k$ patients and each of them has $n_{k}$ service requirements. The number of all patient-related nodes (i.e., all possible pairwise of the patients' locations and their needed services) in the network, namely $n$, is equal to the sum of all required services for all patients (i.e., $n=n_{1}+n_{2}+\ldots+n_{k}$ ). Therefore, the logistics network comprises these patient-related nodes and four other virtual nodes corresponding to the home health 
care company. It should be noted that if a patient needs more than one staff member for a service, the respective service is converted to some virtual services called simultaneous services whose numbers are equal to the number of needed staff members. When we define "virtual nodes" for the initial, end, break, and blood nodes in the network, it means that these nodes are in the same location (i.e., they are not physically different) in our problem definition. Therefore, we should create four nodes to handle the optimization problem. Analogously, when we say "virtual services" and define multiple nodes in the network, it means that the requested service by a patient should be performed by multiple staff members. In fact, we have only one service in this case; however, to handle it through the optimization model, we create multiple nodes called virtual nodes. Obviously, if the initial and end nodes are not physically different, there is no reason to call them virtual. Figure 2 schematically illustrates how we create different nodes in the logistics network of the home health care problem. It should be noted that the initial, end, blood, and break nodes could be physically located in different points. In terms of model construction, there is no restriction that staff must return to $\mathrm{HHC}$ for rest and meals. However, without loss of generality, we assumed that all these virtual nodes are physically the same to make the model simple.

In the case of considering multiple locations for these nodes, the proposed model could be implemented without any amendments in the model in terms of the constraints and the objective function.

\subsection{Problem formulation}

Under the explained assumptions in Subsection 3.1.1, we developed a MILP for the HHCRSP. In the following, all needed indices, parameters, and decision variables together with the mathematical model formulation are explained.

\subsubsection{Index sets}

The index sets of the provided model are defined in Table 5 .

\subsubsection{Parameters}

For the interdependent services $(i, j) \in P^{d}$, the permitted time interval between start times of the required services at nodes $i \in P^{0}$ and $j \in P^{0}$ is bounded between a minimal time distance, $\delta_{i j}^{\min }$, and a maximal time distance, $\delta_{i j}^{\max }$.If $\delta_{i j}^{\max }=\delta_{i j}^{\min }=0$, both services at nodes $i$ and $j$ have to be simultaneously initiated (i.e., if a service requires two staff members, this service is converted to two virtual services called simultaneous services). If $\delta_{i j}^{\max } \geq \delta_{i j}^{\min }>0$, it means that the service at node $j$ should be started no sooner than $\delta_{i j}^{\mathrm{min}}$ and no later than $\delta_{i j}^{\max }$ after starting the service at node $i$. It is worth mentioning that to have a better understanding of interdependent services. We assign larger indices to those nodes whose respective services need to be performed later than other services.

For each node $i \in P^{0}$, a time window $\left[e_{i}, l_{i}\right]$ is

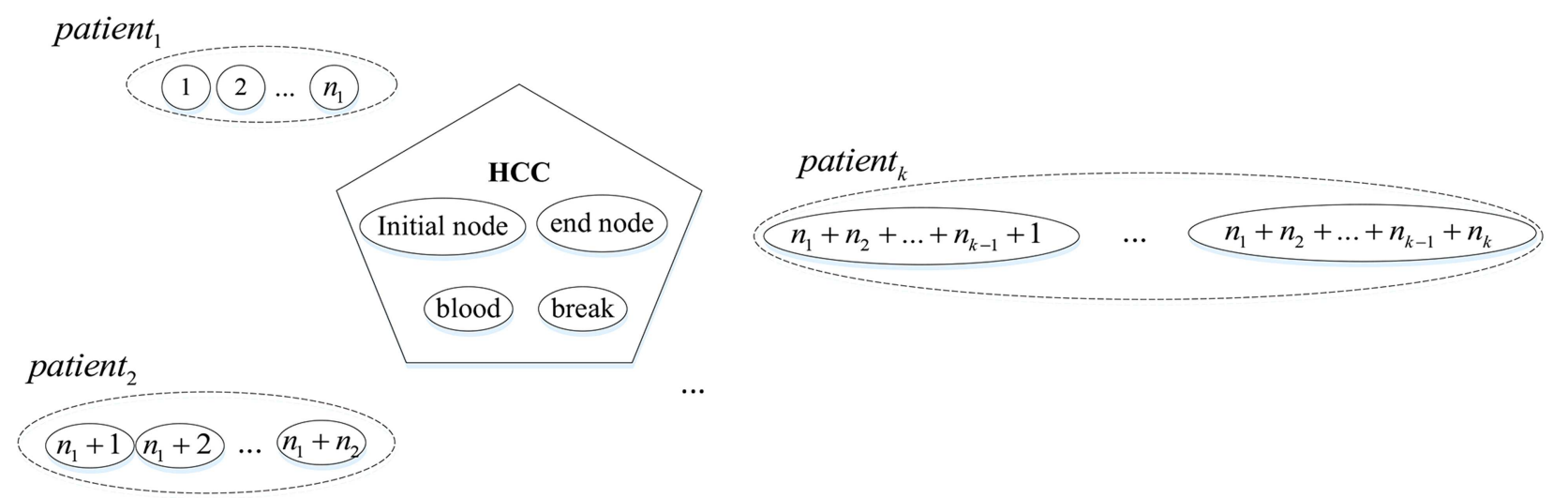

Figure 2. Different nodes in the logistic network.

Table 5. Sets used in the model of HHCRSP.

\begin{tabular}{ll}
\hline $\boldsymbol{P}$ & Index set of all services required by the patients \\
$\boldsymbol{V}$ & Index set of all staff members \\
$\boldsymbol{P}^{\mathbf{0}}$ & Index set of all nodes on the logistics network; i.e., $P^{0}=P \cup 0, n+1$, blood, break \\
$\boldsymbol{P}^{\boldsymbol{d}}$ & Set of pair of nodes requiring timely interdependent services \\
$\boldsymbol{P}^{\text {sim }}$ & Set of pair of nodes requiring simultaneous services \\
$\boldsymbol{P}^{\text {prec }}$ & Set of pair of nodes requiring services with precedence \\
$\boldsymbol{P}^{+}$ & Index set of all patients (nodes) requiring a blood sampling \\
\hline
\end{tabular}


Table 6. Parameters used in the model of HHCRSP.

\begin{tabular}{|c|c|}
\hline$q_{v i}$ & $\begin{array}{l}\text { The qualifications of the staff members, which is equal } 1 \text { if staff member } v \in V \text { is qualified } \\
\text { to perform the required service at node } i \in P^{0} \text { and } 0 \text { otherwise }\end{array}$ \\
\hline$\delta_{i j}^{\min }$ & The minimal time distance between service start times at nodes $i$ and $j$, i.e., $(i, j) \in P^{d}$ \\
\hline$\delta_{i j}^{\max }$ & The maximal time distance between service start times at nodes $i$ and $j$, i.e., $(i, j) \in P^{d}$ \\
\hline$\left[e_{i}, l_{i}\right]$ & Time window of node $i \in P^{0}$ \\
\hline$d u r_{i}$ & The time duration of performing the service required by node $i \in P^{0}$ \\
\hline$d_{i j}$ & Traveling distance between nodes $i \in P^{0}$ and $j \in P^{0}$ \\
\hline$t t_{i j v}$ & Travel time between nodes $i \in P^{0}$ and $j \in P^{0}$ in the route of staff member $v \in V$ \\
\hline$C O C_{j v}$ & $\begin{array}{l}\text { The preferences of patients, which is equal to } 1 \text { iff node } j \in P^{0} \text { prefers staff member } v \in V \text { for } \\
\text { providing its required service and } 0 \text { otherwise }\end{array}$ \\
\hline worktime $_{v}$ & The contract working time of staff member $v \in V$ \\
\hline$[$ estaff,, lstaff. $]$ & Time window of staff member $v \in V$ \\
\hline$T$ & Time horizon \\
\hline$M$ & A very large number, which is equal to the maximum working time allowed to staff \\
\hline$\alpha_{1}, \alpha_{2}, \alpha_{3}, \alpha_{4}$, and $\alpha_{5}$ & The coefficients of the respective terms in the objective function \\
\hline
\end{tabular}

Table 7. Decision variables used in the model.

\begin{tabular}{ll}
\hline & $\begin{array}{l}\text { The routing of the staff members } \\
x_{i j v}\end{array}$ \\
$t_{i v}$ & $\begin{array}{l}\text { if staff } v \in V \text { moves from } i \in P^{0} \text { to } j \in P^{0} \\
\text { for providing the service required bynode } j \\
0 \quad \text { otherwise }\end{array}$ \\
$z_{i}$ & The start time of the required service at node $i \in P^{0}$ provided by staff member $v$ \\
$o v_{v}$ & Tardiness of service required by node $i \in P^{0}$ \\
$l_{-} t w_{v}, u_{-} t w_{v}$ & The overtime of staff member $v \in V$
\end{tabular}

given for the start of the required service. In the case of arriving at the patient location (node $i$ ) sooner than $e_{i}$, the staff member must wait until the Lower Bound (LB) of the time window is reached, otherwise, they can start providing the service. Obviously, there is a penalty if the staff gets the node later than $l_{i}$. For all simultaneous services whose nodes belong to $P^{\text {sim }}$, the services can be started when all needed staff members are available. It is also clear that the time windows for all simultaneous services pertinent to a patient are the same. For all pairwise of services in $p^{p r e c}$, the start times should be arranged taking into account the respective distances $\delta_{i j}^{\mathrm{min}}$ and $\delta_{i j}^{\mathrm{max}}$. The distance between any two nodes $i \in P$ and $j \in P$ is denoted by $d_{i j}$. We assume that the travel time from $i$ to $j$ travelled by staff $v \in V$ is proportional to the distance $d_{i j}$, denoted by $t t_{i j v}$. Brief explanations of all needed parameters are presented in Table 6 .

\subsubsection{Decision variables}

The main decision variable representing staff routing is $x_{i j v} . t_{i v}$ is the start time of the required service performed by staff member $v \in V$ at node $i \in P^{0}$. If the time of providing the service is started with delay (i.e., after $l_{i}$ in the respective time windows), a penalty cost called tardiness value, $z_{i}$, is imposed in the objective function. This cost is calculated by the time span between $t_{i v}$ and $l_{i}$. In the case that staff $v \in V$ works more than his/her contract working time, an overtime value $o v_{v}$ is computed. Finally, if a staff $v \in V$ starts his/her tasks before the LB of his/her desired time window, estaff, and beyond the upper bound of his/her desired time window, lstaff $v$, a penalty function called the violation values, $l_{-} t w_{v}$ and $u_{-} t w_{v}$, areimposed to the objective. $l \_t w_{v}$ is computed using the difference between estaff, and the start time of his/her work and $u_{-} t w_{v}$ is calculated using the distance between the start time of his/her work and lstaff $v$. The decision variables are summarized in Table 7 .

\subsubsection{Objective function}

The companies always try to decrease the costs, increase the quality of the services, and accordingly increase the satisfaction level of the patients and staff 
members. In this paper, we consider five performance measures as follows:

1. The total time traveled by all staff members to provide the required services is given by:

$$
\sum_{v \in V} \sum_{i \in P^{0}} \sum_{j \in P^{0}} t t_{i j v} x_{i j v}
$$

Assuming that staff members have different vehicles with different speeds, the travel time between two nodes depends on the staff who are supposed to provide the required services.

2. The total tardiness in providing services to the patients is given by $\sum_{i \in P^{0}} z_{i}$ where the services start beyond their time windows. In fact, if the staff arrives at the patient's home later than the upper bound of the respective time window, the objective is penalized. Because the salaries of all staff members are assumed to be paid on a daily basis with regard to their contracts, considering no penalty in the objective would be reasonable in the case of the early arrival of the staff members (i.e., before the respective time windows of patients). Moreover, if the waiting of the staff members for providing services leads to overtime, the objective is properly penalized in the third term.

3. The total overtime of the staff members is given by: $\sum_{v \in V} o v_{v}$.

4. The total violation of $\mathrm{CoC}$ is given by:

$$
\sum_{j \in P^{0}} \sum_{v \in V} C o C_{j v} \cdot d u r_{j} \cdot\left(1-\sum_{i \in P} x_{i j v}\right) .
$$

In fact, if a staff member, who is in favor of the respective patient, performs the required services, no penalty is considered in the objective function; otherwise, a penalty equivalent to the duration of the service is imposed into the objective. Taking this term into consideration not only improves the relation between the staff and the patient but also improves the quality of the services.

5. The violation of the staff's time windows is given by:

$$
\sum_{v \in V}\left(l \_t w_{v}+u \_t w_{v}\right) .
$$

In fact, we consider a soft constraint by which all services should be preferably performed within the respective time windows of staff members. Of course, the respective cost for violating these soft constraints could be quite high which means that the constraint is converted to hard ones for some specific cases or patients.
The above cost components could be linearly combined with each other to make a weighted objective function written as follows, where $\alpha_{1}, \alpha_{2}, \alpha_{3}, \alpha_{4}$, and $\alpha_{5}$ are the given coefficients for the respective terms of the objective function:

$$
\begin{aligned}
\min z= & \alpha_{1} \sum_{v \in V} \sum_{i \in P^{0}} \sum_{j \in P^{0}} t t_{i j v} x_{i j v}+\alpha_{2} \sum_{i \in P^{0}} z_{i}+\alpha_{3} \sum_{v \in V} o v_{v} \\
& +\alpha_{4} \sum_{j \in P^{0}} \sum_{v \in V} C o C_{j v} . d u r_{j} \cdot\left(1-\sum_{i \in P} x_{i j v}\right) \\
& +\alpha_{5} \sum_{v \in V}\left(l_{-} t w_{v}+u_{-} t w_{v}\right) .
\end{aligned}
$$

\subsubsection{Problem constraints}

There are two important sets of constraints called staff routing and scheduling. All these constraints in addition to other specific constraints are explained as follows:

- Constraints related to staff routing:

$$
\begin{aligned}
& \sum_{i \in P^{0}} x_{0, i, v}=\sum_{i \in P^{0}} x_{i, n+1, v}=1, \quad \forall v \in V, \\
& \sum_{j \in P^{0}} x_{j i v}=\sum_{j \in P^{0}} x_{i j v}, \quad \forall i \in P, \quad \forall v \in V, \\
& \sum_{v \in V} \sum_{j \in P^{0}} x_{j i v}=1, \quad \forall i \in P^{0}, \\
& x_{i j v} \leq q_{v j}, \quad \forall i, j \in P^{0}, \quad v \in V, \\
& x_{i j v} \in\{0,1\}, \quad \forall i, j \in P^{0}, \quad v \in V .
\end{aligned}
$$

Constraint (2) ensures that the route of each staff member starts and ends in the central depot. Constraint (3) is inflow-outflow conditions or flow conservation constraint, which guarantees that after visiting a node, each staff member must leave the node. This constraint also makes sure that the staff routes remain circular. Constraint (4) indicates that each required service is assigned to exactly one staff member. The domains of the routing variables are determined in Constraint (5), in which a staff $v$ can execute the service required by the node $j \in P$, provided that staff $\nu$ is qualified to perform this service. Such a constraint eliminates many binary variables related to those service requirements that are incompatible with the qualification of a staff member. Constraint (6) enforces integrality restrictions on the binary variables.

- Constraints related to staff scheduling:

$$
\begin{gathered}
t_{i v}+d u r_{i}+t t_{i j v} \leq t_{j v}+M\left(1-x_{i j v}\right), \\
\forall i \in P^{0}, j \in P^{0}, v \in V,
\end{gathered}
$$




$$
\begin{aligned}
& t_{i v} \geq e_{i} \sum_{j \in P} x_{j i v}, \quad \forall i \in P^{0}, v \in V \\
& t_{i v} \leq l_{i} \sum_{j \in P} x_{j i v}+z_{i}, \quad \forall i \in P^{0}, \quad v \in V \\
& t_{j v_{2}}-t_{i v_{1}} \geq \delta_{i j}^{\min }-M\left(2-\sum_{k \in P^{0}} x_{k i v_{1}}-\sum_{k \in P^{0}} x_{k j v_{2}}\right) \\
& \forall(i, j) \in P^{d}, \quad v_{1}, v_{2} \in V, \\
& t_{j v_{2}}-t_{i v_{1}} \leq \delta_{i j}^{\max }+M\left(2-\sum_{k \in P^{0}} x_{k i v_{1}}-\sum_{k \in P^{0}} x_{k j v_{2}}\right) \\
& \forall(i, j) \in P^{d}, \quad v_{1}, v_{2} \in V, \quad \forall i \in P^{0}, \quad v \in V . \\
& t_{i v}, z_{i}, o v_{v} \geq 0, \quad(10
\end{aligned}
$$

Constraint (7) determines the start times of the services with respect to the duration of performing the services and the travel times between two nodes. In fact, this constraint indicates that the start times of the services along the route of a staff member are strictly increasing. Constraints (8) and (9) control the start times of the services that should comply with the time windows of the patients. Each patient has the propensity to be served in a given time window. It should be noted that the LBs of the nodes time windows have to be satisfied (i.e., they are considered as hard constraints) while the upper bounds could be violated (i.e., they are considered as soft constraints). In other words, if the upper bound of the time window of node $i, l_{i}$, is overshot, a tardiness, $z_{i}>0$, is added to the starting time of providing the required service in that node. It is worth noting that in some specific requirements (e.g., an injection that should be performed within a specific time interval) the upper bound should be hard; however, for many other requirements of patients it would be soft. Temporal interdependences are managed by Constraints (10) and (11) in which the bounds of time distances between two interdependent nodes must be satisfied. One of the most important interdependences is "simultaneousness", in which the minimum and maximum time distances get zero values. Finally, non-negative Constraint (12) control non-negativity restrictions on scheduling, tardiness, and overtime variables.

- Other constraints related to increasing efficiency of the problem:

$$
\begin{aligned}
& t_{n+1, v}-t_{0, v} \leq T, \quad \forall v \in V, \\
& o v_{v} \geq t_{n+1, v}-t_{0, v}-\text { worktime }_{v}, \quad \forall v \in V .
\end{aligned}
$$

Constraint (13) controls the total working time for a staff member which should not be larger than the time horizon. The total overtime of each staff member with respect to his/her contract working time is also calculated in Constraint (14). In fact, if a staff waits until the time window of a patient opens up, the home health care organization usually has to compensate for this waiting time by considering overtime for providing other services.

In this paper, we assumed that the initial, end, blood, and break nodes are located in the home health care center. As it was mentioned, we can consider multiple locations as central depots without any amendments in the optimization model in terms of the constraints and the objective function. In other words, we should specify the set of central depots for all staff members, which could be their homes, in the preprocessing phase and create needed virtual nodes for these new central depots. The respective constraints such as the Constraints (2), (13), and (14) in the optimization model have a minor change in indexes (e.g., $x_{0, i, v}$ is replaced with $x_{h o m e \_v, i, v}$ in which "home_v" is the initial node number for staff member $\nu)$. It means that all the respective changes should be performed in the preprocessing phase and no changes happen in the number or the form of constraints and objective functions.

$$
\begin{aligned}
& t_{0, v} \geq \operatorname{estaf} f_{v}-l \_t w_{v}, \quad \forall v \in V, \\
& t_{n+1, v} \leq \text { lstaf } f_{v}+u_{-} t w_{v}, \quad \forall v \in V .
\end{aligned}
$$

Constraints (15) and (16) take the desired time windows of staff members into account in a soft way. This means that if the time window for a staff member is violated, a penalty function equivalent to the sum of $l_{-} t w_{v}$ and $u_{-} t w_{v}$ are added to the objective function.

$$
\begin{aligned}
& 1+\left(l_{\text {blood }}-t_{i, v}\right) \leq M\left[\sum_{j \in P} x_{j, \text { blood }, v}+1-\sum_{j \in P^{0}} x_{j i v}\right] \\
& \forall i \in P^{+}, \quad \forall v \in V, \\
& t_{\text {blood }, v} \leq l_{\text {blood }} \cdot \sum_{j \in P^{0}} x_{j, \text { blood }, v}, \quad \forall v \in V \\
& t_{\text {blood }, v} \geq t_{i, v}, \quad \forall i \in P^{+}, \quad v \in V .
\end{aligned}
$$

The above constraints are pertinent to the blood samples. Based on medical and biological reasons, all blood samples should be returned to the home health care center within a predefined time. If there is any node requiring the blood sampling in the route of a staff member, he/she has to return it to the virtual blood node before the upper bound of the time window corresponding to that node without any tardiness. In summary, Constraint (17) means that whenever a staff 
member takes a blood sample at least in one node $i \in P^{+}$, then he/she must visit the virtual destination nodeblood before $l_{\text {blood }}$. Constraint (18) assures that the staff $v \in V$ returns to this virtual node before the upper bound of its time window. Constraint (19) ensures that the nodeblood is visited after the last blood sample has been taken. For more details, one could refer to Elbenani et al. [9].

$$
\sum_{i \in P^{0}} x_{i, b r e a k, v}=1, \quad \forall v \in V .
$$

Constraint (20) assures that every staff member must visit the break node, which is defined as a virtual node (it could be the same as a home health care center in reality). As we explained, there is no limitation to consider different break nodes for each staff member. In particular, the proposed optimization model could be simply customized to new problems with multiple nodes for break times. Each break node has its own time windows and time duration, which are exactly the same service nodes that should be specified by the modeler before making the optimization model. In our case study, we only considered one as a single breakpoint with a specific time window for all staff members, which can be easily extended to multiple nodes in other problems.

Bertels and Fahle [18] demonstrated that the routing and scheduling problem in home health care without considering temporal interdependencies is also NP-hard. In our proposed model, there are more variables and linear constraints compared to other analogous models, which make our model even more complex. Therefore, to find a good solution (i.e., nearoptimal solution), we need an appropriate solution scheme. A major focus of this paper is to propose a solution scheme based on GA, which can appropriately solve our NP-hard model constructed for real-life applications.

\section{GA as the solution scheme for solving the HHCRSP}

Using exact methods such as those provided in GAMS to find the optimal solution for large instances is computationally expensive. This means that the optimization problem is intractable for exact solutions by conventional methods and the optimal solution cannot be reached within a reasonable time. In this section, we applied a GA-based algorithm, which could handle the respective models made for real-life problems. GA is a bio-inspired, probabilistic search method based on the natural selection method which was initially developed by Holland [38]. Gen et al. [39] proved that GA would be a proper approach to acquire near-global solutions. In the following, we explain how to apply the GAbased algorithm for solving the routing and scheduling problem. We made some changes to the traditional GA in order to resolve the model within a reasonable time and produce a more qualified solution.

\subsection{Solution representation}

The most important part of using metaheuristics as a solution in the practical optimization model is to provide proper constraints handling to satisfy them in an appropriate way. To handle the constraints and to implement the coding procedures, three matrixes must be defined. In other words, to consider the assignment and scheduling of services in the HHCRSP, each possible solution (chromosome) is introduced by three matrices: (1) assignment matrix (A), (2) scheduling matrix $(\mathrm{S})$, and (3) the matrix representing the start time of providing services for each staff member $\left(T_{0}\right)$. The procedures for making these matrices are described as follows:

a. The initial part of a chromosome is a $1 \times n$ matrix, in which $n$ is the number of all service requirements and each node is randomly assigned to a staff member, which the qualifications of the staff must be compatible with the respective service requirements. (i.e., each member in the matrix $A$ specifies the staff member who should provide the corresponding required service). Each entry is selected based on Uniform (1, cardinality $\left.\left(\left\{v \mid q_{v i}=1\right\}\right)\right)$;

b. The second part of a chromosome is a by $n+$ $2 v$ matrix whose members are determined as a permutation from 1 to $n+2 v$. In fact, this part of the solution determines the sequence of visiting the patients by staff members. It should be noted that in such a matrix, the nodes corresponding to blood and break are used as virtual nodes, which are also defined and replicated according to the number of staff members (i.e., each member in matrix $S$ specifies providing a required service or going to a virtual node);

c. The final part is a $1 \times v$ matrix representing the times that the staff members start their works. For this matrix, a random continuous value is uniformly chosen between 0 and $T$.

To make the solution representation more clear, we present an example by which the process of finding all routes of staff members is explained. The assignment matrix, scheduling matrix, start time matrix, routes, and schematic network of the feasible solution for the example are denoted in parts of Figure 3, respectively. As it is illustrated in this figure for $n=9$ and $\nu=$ 3 , there are fifteen nodes in which 10,11 , and 12 represent the virtual nodes blood that correspond to staff members 1, 2, and 3, respectively. The same explanation can be repeated for 13,14 , and 15 as the virtual nodes break. Therefore, each of these virtual 


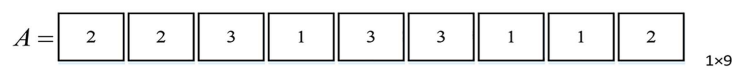

(a)

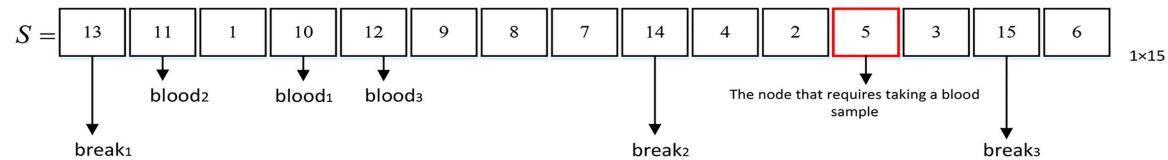

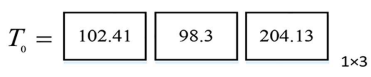

(c)

(b)

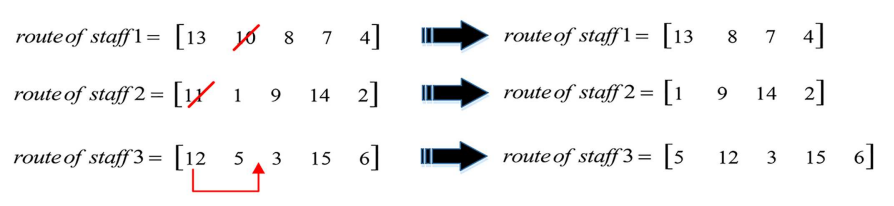

(d)

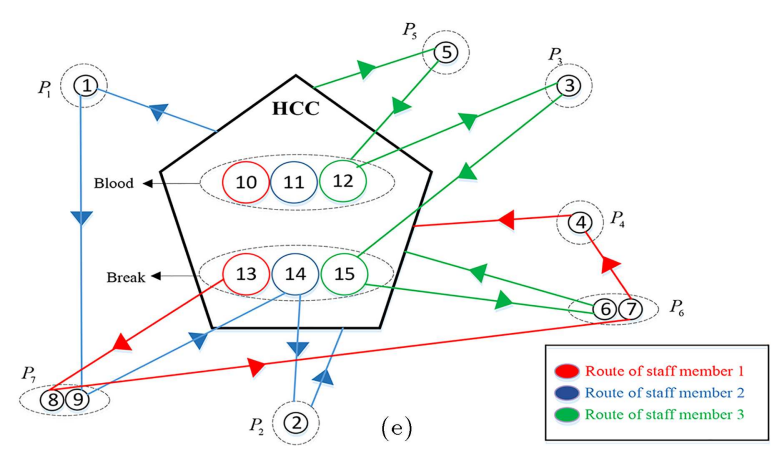

Figure 3. The Solution representation for the explained example.

nodes is assigned to one staff member, that is, a staff member assigned to a virtual node is not allowed to go to other virtual nodes.

In this example, we end up with three routes as there are three staff members. As it is demonstrated in part d of Figure 3, the first node would be 13 as matrix $S$ is started with this node, which solely corresponds to the first staff member. Therefore, the starting point for the first staff member is node 13. The next node after node 13 in matrix $S$ is virtual node 11, which solely corresponds to the second staff member, therefore it is put in the second route. The third node in matrix $S$ is 1, which has to be assigned to the second staff member based on matrix $A$. Therefore, this node is placed in the second route after the virtual node 11 . The next node based on matrix $S$ is 10 , which corresponds to the virtual node blood for the first staff member. As a result, considering the sequences in matrices $S$ and $A$, nodes 8,7 , and 4 also correspond to the first route, nodes 14,9 , and 2 belong to the second route, and finally, nodes 12, 5, 15, 3, and 6 are assigned to the third route. To have feasible routes, some rerouting procedures should be performed. Since there is not any node that requires a blood sampling, node $10\left(\right.$ blood $\left._{1}\right)$ is removed from the first route. The same thing occurs for the virtual node $11\left(\right.$ blood $\left._{2}\right)$ in the second route. Moreover, as returning to the blood node in each route should take place after visiting all the nodes with blood sampling requirements, the sequence of 5 and 12 must be reversed. To make it a general rule, if the node blood is located after all nodes that require a blood sampling, this route is feasible, otherwise, the node blood must be put right after the last assigned node in which the blood sample needs to be taken.

\subsection{Decoding}

The whole process of decoding is schematically illustrated in Figure 4. As obvious in this figure, after generating the three matrices and the feasible routes which have been fully explained in the previous section, the start times of all required services could be calculated. Using these start times, the objective function of our proposed model given by Eq. (1) is computed. In addition, two penalty functions corresponding to soft Constraints (13) and (18) are estimated based on both the start times of required services and the start times in matrix $T_{0}$. All this information can be used to find the fitness function value for the respective solution (chromosome).

The following equations are used to find the penalties:

$$
\begin{aligned}
& p n f_{1}=\sum_{k=1}^{v} \max \{t(n+1, k)-t(0, k)-T, 0\}, \\
& p n f_{2}=\sum_{k=1}^{v} \max \left\{t\left(\text { blood }_{k}, k\right)-l\left(\text { blood }_{k}\right), 0\right\},
\end{aligned}
$$

where $p n f_{1}$ and $p n f_{2}$ are the total violation of constraints related to the time horizon (Constraint (13)) and delays in returning the blood samples to the blood node for all staff members (Constraint (18)), respectively. The fitness function is also calculated using the following formula:

Fitness function value $=$ objective function value

$$
+\alpha\left(1+\gamma\left(p n f_{1}+p n f_{2}\right)\right)
$$

where $\gamma$ is a predefined coefficient that should be determined by decision-maker/s. 


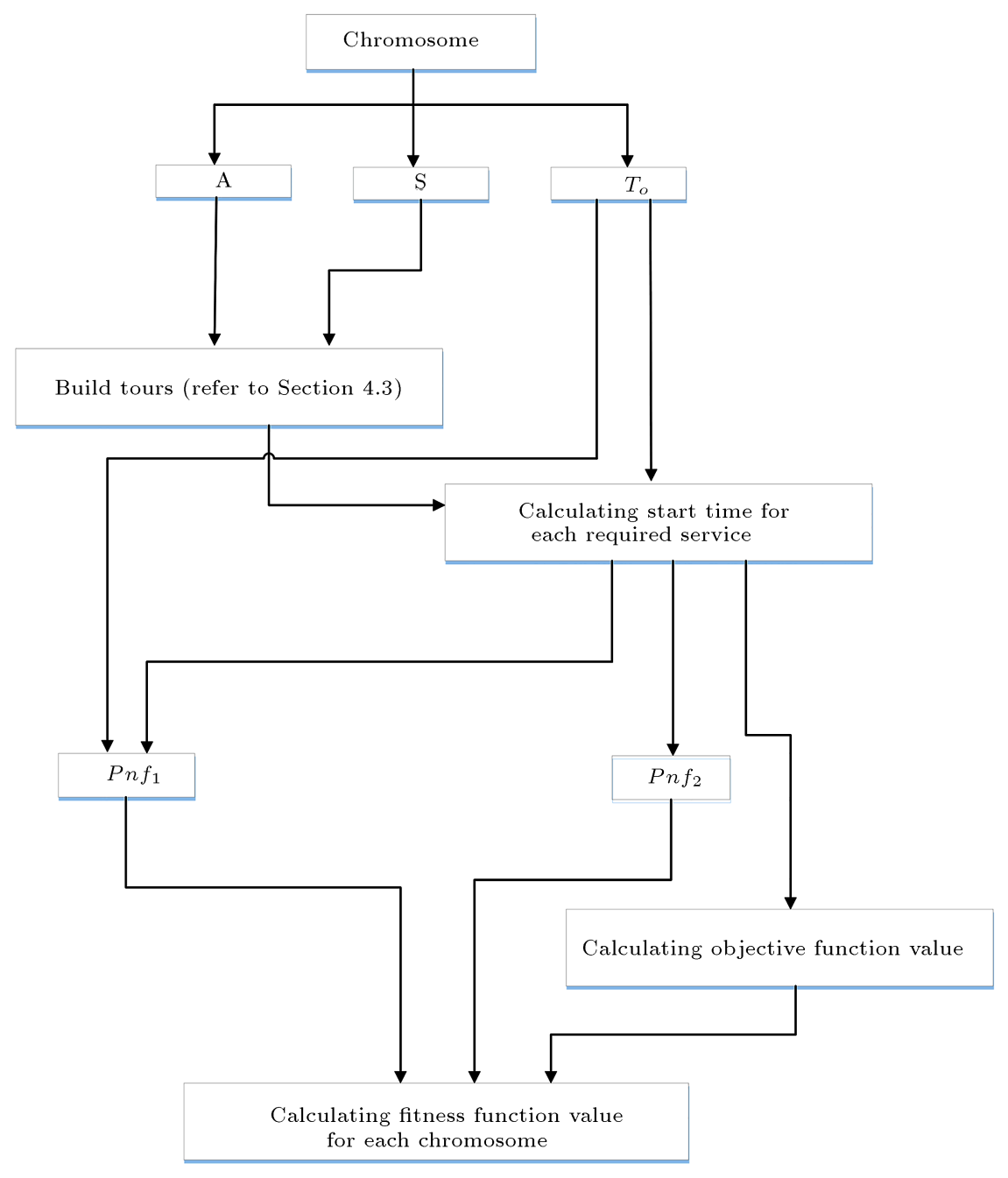

Figure 4. Flowchart of the decoding process.

The fitness function, the sum of the objective and penalty functions determined by the degree of infeasibility, is the only criterion by which we could choose the next generation and find an appropriate solution. It is worth mentioning that any chromosome in the current generation (parents) with the lowest/highest fitness function in a minimum/maximum objective is not necessarily transferred to the next generation.

\subsection{Proposed operators}

The solution quality and the speed of convergence depend on the algorithm parameters. These parameters include:

1. Number of generations, which affects the solution quality;

2. Population size, which is effective for the search in the solution space;

3. Crossover rate, which determines the probability of performing the crossover operator on a chromosome;
4. Mutation rate, which specifies the probability of performing the mutation operator on a chromosome to avoid getting trapped in a local optimal solution.

In our genetic-based solution scheme, three crossover and mutation operators are used. In fact, to find more feasible solutions through crossover and mutation, these operators run for each part individually. Without the mentioned partitioning, we could not find a feasible and good solution at a reasonable time for our problems. In the following, all these operators are explained in detail.

\subsubsection{Crossover operator}

The crossover operator plays a major role in creating successive generations. In fact, the role of this operator is to locally search the solution space and to find better offsprings in a limited space. The probability of crossover $\left(p_{c}\right)$ is a predefined parameter that should be determined based on the problem size. Choosing a suitable crossover operator for generating offsprings is quite dependent on the structure of the chromosome. 
As mentioned earlier, each chromosome contains three matrices, where the crossover operator must be performed on all three parts in a different way in order for offsprings to become feasible. These are explained in details as follows:

a. The appropriate crossover operator for $A$ is a uniform crossover. In the uniform crossover, after producing a mask matrix with zero/one genes, each gene of the offspring is chosen with respect to the corresponding genes in the mask and the respective parents. If the value of the gene in the mask matrix is equal to zero, the respective genes in both parents should be replaced, otherwise, the genes for offsprings are the same as the parents. Figures 5 and 6 demonstrate how the crossover operation

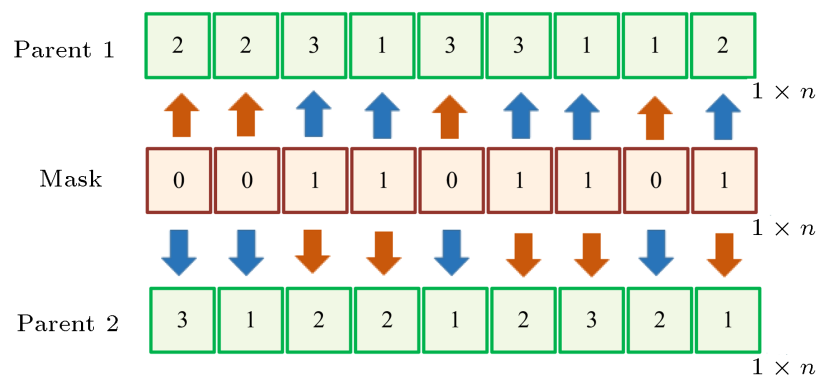

Figure 5. Mask process of producing both offsprings.

\begin{tabular}{l|l|l|l|l|l|l|l|l|l|l|} 
Offspring 1 & 3 & 1 & 3 & 1 & 1 & 3 & 1 & 2 & 2 \\
\hline
\end{tabular}

Figure 6. Offsprings after crossover operation for matrix A. is performed on the matrix $A$ with nine service requirements. As it is schematically illustrated, the first gene in the mask matrix is zero, leading to replacing the respective genes of the parents for finding the first genes of two offsprings. This has to be repeated for all eight remaining genes to produce the offsprings;

b. The appropriate crossover operator for $S$ is a type of single-point crossover. However, this crossover operator must be properly adjusted such that it could consider the permutation structure to avoid any gene repetition. This type of crossover is called as the adjusted single-point crossover operator. In the first step, two chromosomes (parents) are split using a randomly selected point, namely the crossover point. All genes in both offspring before this point are the same genes of their parents; However, after this point, the genes of the offspring definitely change. Finally, for making the second part of the first offspring, we should go through all genes in the second parent, find all non-repeated genes in the first part of the first parent, and put them in the second part of the first offspring without changing their orders. The second offspring is made in a similar way. Given that the proposed crossover operator is executed under the assumption that the permutation attribute in the matrix $S$ and the difference between all genes are considered, it is obvious that the solution in the offspring is also feasible. The procedure of producing offsprings in the matrix $S$ with nine service requirements and three staff members is depicted in Figures 7 and 8;

c. The appropriate crossover operator for $T_{0}$ is a continuous type of uniform crossover in which a $1 \times v$ matrix $(\alpha)$ including $v$ uniformly random numbers

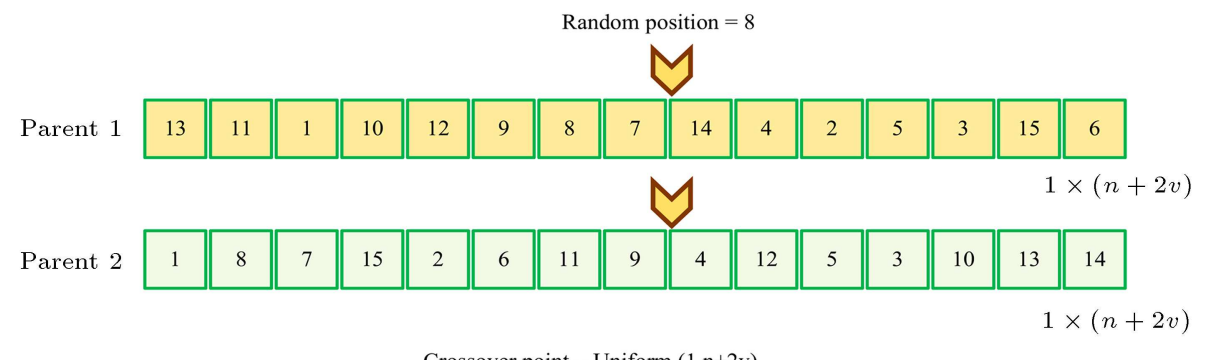

Figure 7. The proposed crossover point for matrix $S$.

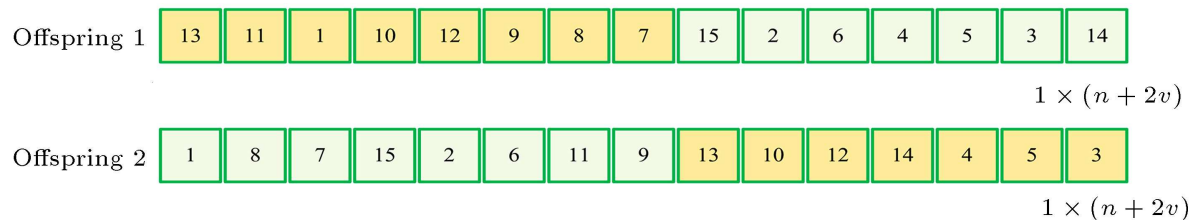

Figure 8. Offsprings after crossover for matrix $S$. 


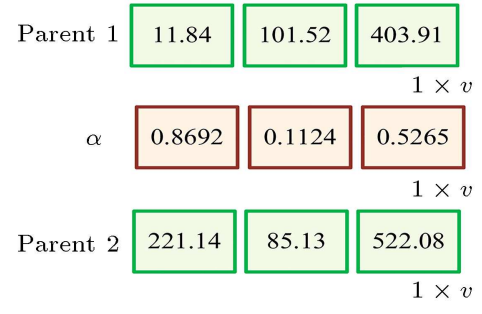

Figure 9. The proposed crossover for matrix $T_{0}$.

between 0 and 1 is initially generated. Each gene should be computed using a convex combination of the respective gene in the generated matrix and the corresponding genes in two selected parents. The process of producing offsprings where there are three staff members is illustrated in Figures 9 and 10 .

\subsubsection{Mutation operator}

The main role of this operator is to prevent getting trapped in the locality. In fact, this operator performs random perturbations to selected solutions leading to a gene sudden alteration by which the solution space would be remarkably dispersed. In other words, the search space using the operator would be more extended, that is, new information that is more valuable could be injected into the population. However, this operator should have quite a lower probability $\left(p_{m}\right)$ compared with the crossover operator due to the issue of divergence. Here, we explain in detail how the mutation operator is performed for three solution matrices:

a. The appropriate mutation operator for $A$ is a type of uniform mutation. In the first step of this operator, a $1 \times n$ matrix including $n$ random numbers which are uniformly distributed between zero and one is produced. All the genes in a selected parent smaller than a predefined rate (e.g., $\mu=0.1$ ) are candidates to be modified. The respective candidates should be replaced with new staff members who are randomly chosen from a set $\left[1,|V|_{\text {qualified }}\right]$, where $|V|_{\text {qualified }}$ is the total number of all qualified staff members for the pertinent services in the candidate genes. This causes the constraint related to the qualification of staff members (constraint (5)) to

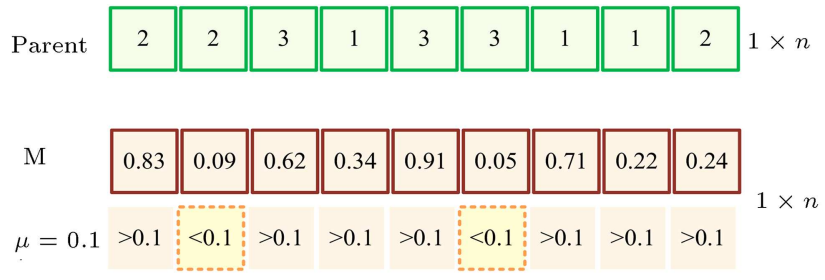

Figure 11. The proposed mutation point for matrix $A$.

\begin{tabular}{l|l|l||l||l||l|l|l|l|l|l|}
\hline Offspring & 2 & 1 & 3 & 1 & 3 & 2 & 1 & 1 & 2 \\
\hline
\end{tabular}

Figure 12. Offsprings after mutation for matrix $A$.

be satisfied and the feasibility is therefore guaranteed. Figures 11 and 12 demonstrate the procedure of the mutation operator for the matrix $A$ with nine service requirements. It should be mentioned that the qualified staff members for the second and sixth genes (candidate genes) are $\{1,2\}$ and $\{2,3\}$, respectively;

b. Regarding the particular structure of the scheduling chromosome, implementing a mutation operator for $S$ consists of two vital phases: (1) randomly choosing two elements of the matrix $S$ and (2) randomly selecting one of the operations among swap, reversion, and insertion. In other words, given two selected genes in the respective matrix, a random integer number between 1 and 3 is selected (1, 2, and 3 are related to swap, reversion, insertion operators, respectively). In the swap operation, the two genes should be replaced. In the reversion operation, all genes between the two selected genes are entirely reversed. In the insertion operation, the larger gene should be shifted to right after the smaller gene;

c. The appropriate mutation operator for $T_{0}$ is a type of uniform mutation, in which the times for starting the services for each staff member are changed such that it does not violate the time horizon. The pseudo-code is demonstrated in Figure 13 where $\lambda$ is a predefined coefficient that determines the maximum range of variations in each gene.

\subsubsection{Selection operator}

To form the next generation, the numer " $p$ " of the

$$
\begin{aligned}
& \text { for } i=1 \text { to } v \\
& \left.\qquad \begin{array}{l}
\text { offspring } 1(i)=\alpha(i) \cdot \text { parent } 1(i)+(1-\alpha(i)) \text {. parent } 2(i) \\
\text { offspring } 2(i)=\alpha(i) \cdot \text { parent } 2(i)+(1-\alpha(i)) \cdot \text { parent } 1(i)
\end{array}\right\} \\
& \text { end for }
\end{aligned}
$$

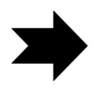

Offspring 1

Offspring 2
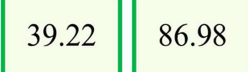

459.86
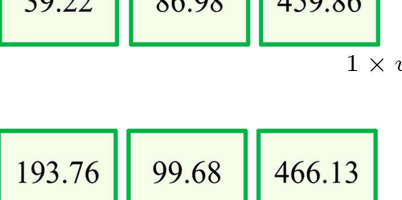

466.13

Figure 10. Offsprings after crossover for matrix $T_{0}$. 


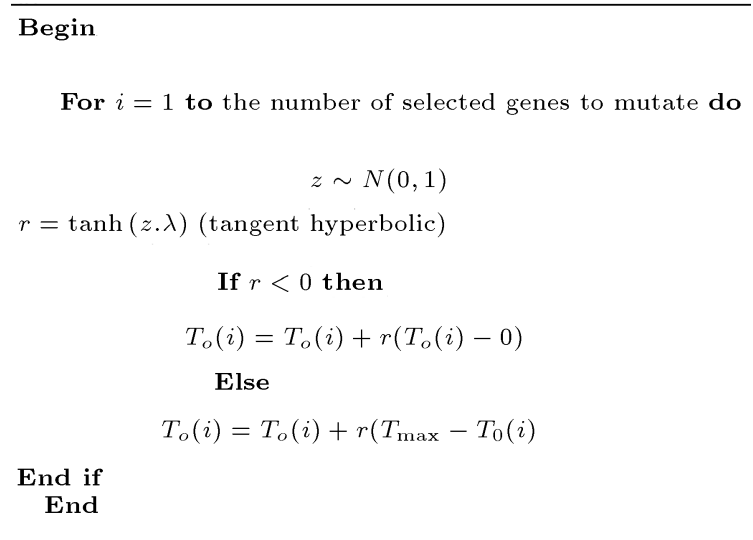

Figure 13. Pseudo-code of mutation operator for $T_{0}$.

best solutions amongst the previous generation and the new offsprings must be retained. Selection is also the process of choosing all pair-wires of chromosomes on which the crossover operators are performed. The purpose of defining such an operator is to select parents with higher fitness functions that could produce better offsprings. In this paper, the Roulette wheel method has been used as the selection operator.

\section{Computational experiments}

Since there are usually no accepted benchmark sets in the literature or the respective data sets are not fully available, this study cannot provide a comparison with real or benchmark examples. This would be also a common approach in many recently published papers to use make-up examples to investigate the efficiency of their proposed models and solution schemes. One can refer to [13] for observing the list of these papers. Here, we initially start with a simple example to evaluate the validity of the model and the proposed solution scheme. Then, in the second part of the experiments, we generate many instances with different sizes (i.e., small- to large-sized instances) to show the efficiency of the solution method in different problems. The proposed model and the solution scheme were implemented in GAMS and MATLAB R 2013b, respectively. In addition, all calculation experiments were performed on a laptop computer with $2.40 \mathrm{GHz}$ Intel Core i5 CPU and 4 GB RAM memory.

\subsection{The first part of the experiments (validity of the model and the solution scheme)}

Assume that there is a problem consisting of a home health care center and six real patients (i.e., patients locations) in a logistics network of $1000 \times 1000$ distance units (The travelling distances are calculated using Euclidean way). These patients are categorized into two groups: (1) The first group denotes the patients requiring single services (i.e., the first four patients)
Table 8. Specifications of the patients.

\begin{tabular}{cccc}
\hline \multirow{2}{*}{ Node $\boldsymbol{i}$} & $\begin{array}{c}\text { Duration } \boldsymbol{d u r}_{\boldsymbol{i}} \\
\text { (time unit) }\end{array}$ & \multicolumn{2}{c}{ Time window } \\
\cline { 3 - 4 } & & $\boldsymbol{e}_{\boldsymbol{i}}$ & $\boldsymbol{l}_{\boldsymbol{i}}$ \\
\hline 0 & 0 & 0 & 600 \\
1 & 40 & 185 & 348 \\
2 & 30 & 250 & 227 \\
3 & 30 & 115 & 145 \\
4 & 50 & 132 & 391 \\
5 & 30 & 258 & 314 \\
6 & 30 & 258 & 314 \\
7 & 30 & 152 & 350 \\
8 & 30 & 152 & 350 \\
9 & 15 & 0 & 600 \\
blood & 5 & 0 & 280 \\
break & 25 & 370 & 500 \\
\hline
\end{tabular}

and (2) The latter refers to the rest of the patients who require two interdependent services. Set $P$ includes all patient-related nodes $(1,2, \cdots, 8)$, in which nodes 1,2 , 3 , and 4 are pertinent to the first four patients requiring single service, nodes 5 and 6 are associated with the fifth patient requiring two simultaneous services $\left(P^{\text {sim }}=\{(5,6)\}\right)$, and two other nodes 7 and 8 are associated with the sixth patient requiring two services with fixed precedence $\left(P^{\text {prec }}=\{(7,8)\}\right)$. Thus, set $P^{0}=\bigcup P\{0,9$, blood, break $\}$, where 0,9 , blood, break are four virtual nodes corresponding to the central depot. As a result, there are 12 nodes including the virtual nodes in this network. In addition, only one patient requires a blood sampling service (set $P^{+}=$ $\{4\})$. The duration of time for providing services to patients and time windows are demonstrated in Table 8. The time distances between all pair-wires of service requirements are specified as follows. The required services by nodes 7 and 8 (i.e., patient 6 ) must be started within a time interval of $\left[\delta_{7,8}^{\min }=15, \delta_{7,8}^{\max }=40\right]$. Since the required services by nodes 5 and 6 (i.e., patient 5 ) must be started simultaneously, then the time distances $\delta_{5,6}^{\min }, \delta_{5,6}^{\max }, \delta_{6,5}^{\min }$ and $\delta_{6,5}^{\max }$ are set to zero. The rest of the minimal and maximal time distances in the off-diagonal members of the matrices are fixed to $-600(-T)$ and $600(T)$, respectively, while all diagonal members are set to zero (i.e., $\delta_{i, i}^{\min }=0$ and $\delta_{i, i}^{\max }=0$ ).

Moreover, the qualifications of three staff members $V=\{1,2,3\}$ based on different requirements of the nodes, conversion coefficient of traversed distance to travel time $\left(f_{v}\right)$, work time, and the desired time window are illustrated in Table 9 . All members of the priority matrix $\mathrm{COC}_{i v}$, which indicates the $\mathrm{CoC}$ between all pairwise of nodes and staff members, are set to zero except for $\mathrm{COC}_{3,2}$ which is set to one. 
Table 9. Specifications of staff members.

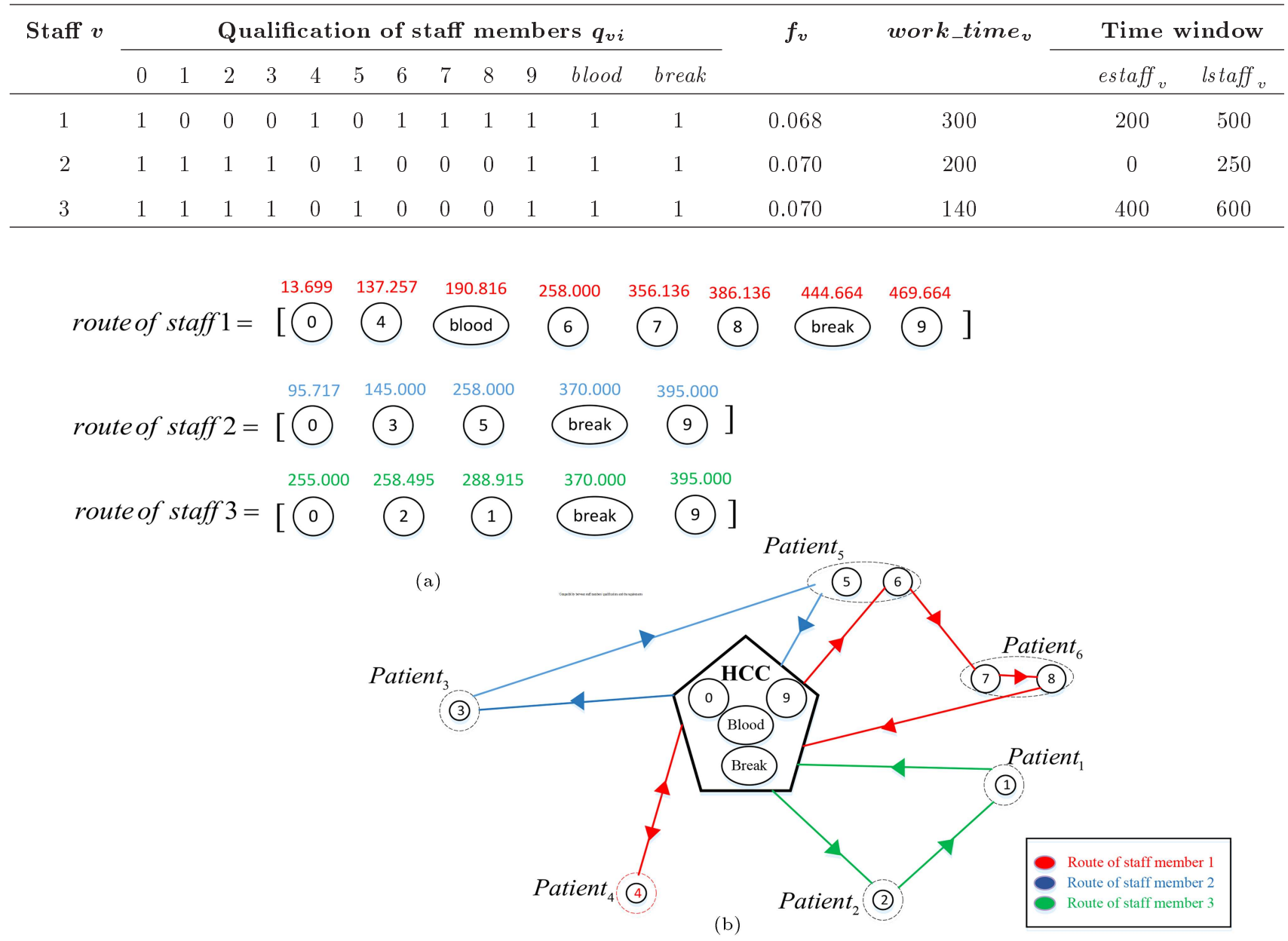

Figure 14. Optimal solution of the explained example.

Furthermore, all the respective coefficients pertinent to performance measures in the objective function are equal to each other (i.e., $\alpha_{1}=\alpha_{2}=\alpha_{3}=\alpha_{4}=\alpha_{5}=$ $\left.\frac{1}{5}\right)$. The parameter $M$ is also assumed to be equal to 600 .

The optimal solution for this example is represented in Figure 14, where the path of each route and the schematic view of the routes in the whole network are depicted in Figures 14(a) and (b), respectively. The start time of performing the required service at node $i$ provided by staff member $v,\left(t_{i v}\right)$, has been specified at the top of each node (part (a)). As observed, the simultaneity of the services required by nodes 5 and 6 and the precedent relationship between the services required by nodes 7 and 8 are met. The optimal value of the objective function is 174.852 , the total distance traversed by all staff members is 308.943 time units, the total tardiness occurred when providing the service requirements is 73.767 time units, and the total overtime of all staff members is 135.248 time units.

It can be observed that most of the time windows of patient-related nodes are fulfilled except that the ser- vice requirements of nodes 2,7 , and 8 start with a delay of $31.495,6.136$, and 36.136 time units, respectively. It is worth mentioning that no staff member arrives at a node before opening the time windows of the patients. Thus, if there are no interdependent services in two nodes, the service requirement is performed immediately after the arrival of staff members at a patients home. In addition, staff members 1 and 2 must work 35.965 and 99.283 time units over their contract working time while the overtime for staff member 3 is zero. Also, the required service at node 3 is provided by staff member 2, that is, the respective COC is satisfied. Therefore, the fourth performance measure becomes zero. Moreover, only the LB of the desired time window for staff member 2 and the upper bounds of time windows determined by staff members 1 and 3 are met.

A similar solution is also obtained using GA for the above example that verifies the validity of the proposed solution scheme. Figure 15 demonstrates the trend of convergence of GA, which ends up with the same objective function of the exact method. 


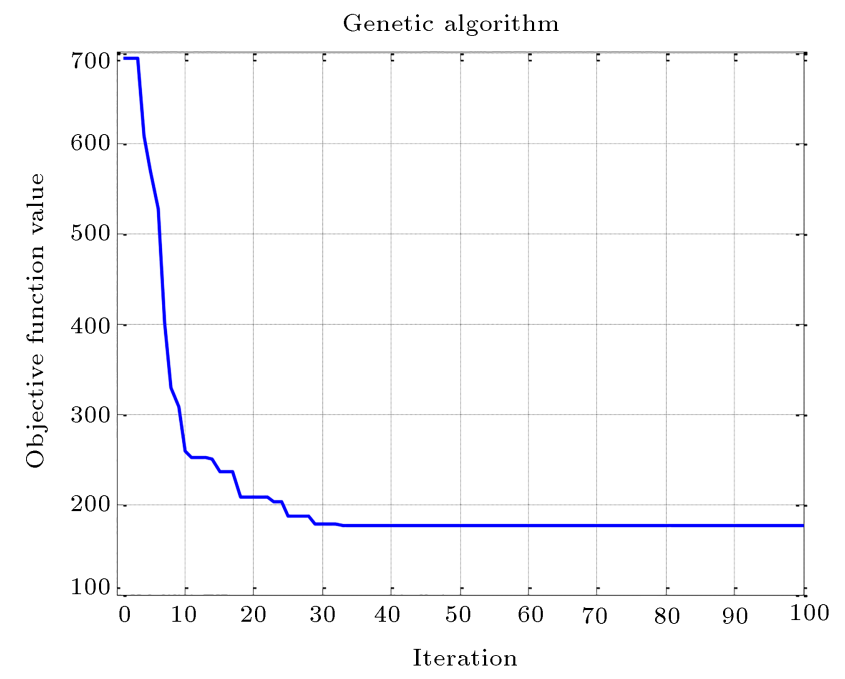

Figure 15. Convergence diagram of the example.

\subsection{The second part of experiments (comparing efficiency of $G A$ in different problems)}

\subsubsection{Description of test instances}

We employ six sets of randomly generated instances with different characteristics (refer to Table 10). For each of these sets, five instances with different values in the nodes coordinates, qualification matrix, and so on are created. It should be mentioned that the traveling distances between all pairwise nodes are generated using a uniform distribution function ranging between 0 and 2000, that is, all generated nodes are located in an area of $2000 \times 2000$ distance units.

To achieve the matrix of travel times, the distance matrix is determined by Euclidean distance using the coordinates of the nodes in the network. Therefore, the travel times can be formulated as $t t_{i j v}=f_{v} \cdot d_{i j}, \forall i, j \in$ $P^{0}, \forall v \in V$, in which $t t_{i j v}$ is the travel time between nodes $i$ and $j$ of staff member $\nu, d_{i j}$ is the traveling distance between these nodes, and $f_{v}$ is the conversion ratio of distance to travel time (speed inversion). It should be noted that the amount of $f_{v}$ really depends on the fleet types of vehicles. We do not distinguish between the five predefined performance measures and the weights for all sub-goals in the objective function are assumed to be equal. All the respective parameters used in our proposed model are demonstrated in Table 11.

To set the parameters of each instance, the random numbers should be generated within the intervals specified in Table 11 for all parameters. For example, a possible value for $\delta_{i j}{ }^{\max }$ is obtained by summing two random values generated within two time intervals $[0,70]$ and $[5,50]$. In addition, the maximum difference between the upper and LBs for the time window of each patient-related node is assumed to be 200 time units.

\subsubsection{Numerical results of the exact solution of the optimization model}

To investigate the performance of our model in which there is one node for each service required by the patient (we call it model 1), we implemented the model in which there is only one node per patient (we call it model 2). The second model is used by Mankowska et al. [7]. It is quite obvious that the problem dimension in model 2 is significantly smaller than the first model, especially for large-scale problems. For example, assume a problem instance with seven real patients and three staff members in which five patients require a single service and two others require interdependent services. Using the first model, the network ends up with thirteen nodes (i.e., seven nodes for real patients and two nodes for virtual patients, and four nodes for virtual nodes, which finally correspond to the home health care center). However, in the case of using the second model, the total nodes including virtual nodes in the example become eleven (i.e., seven nodes for patients and four nodes for virtual nodes corresponding to the home health care center). It should be noted that as the number of patients and the number of their required services increase, the difference in the number of nodes between the two models would significantly rise.

Table 10. Specifications of considered instances sets.

\begin{tabular}{ccccc}
\hline $\begin{array}{c}\text { Instances } \\
\text { set }\end{array}$ & $\begin{array}{c}\text { Number of } \\
\text { patients }\end{array}$ & $\begin{array}{c}\text { Number of } \\
\text { patients requiring } \\
\text { interdependent services }\end{array}$ & $\begin{array}{c}\text { Number of } \\
\text { nodes along } \\
\text { with the } \\
\text { virtual nodes }\left(\left|\boldsymbol{P}^{\mathbf{0}}\right|\right)\end{array}$ & $\begin{array}{c}\text { Number of } \\
\text { staff members } \\
(|\boldsymbol{V}|)\end{array}$ \\
\hline$A$ & 3 & 1 & 8 & 2 \\
$B$ & 7 & 2 & 13 & 3 \\
$C$ & 10 & 3 & 17 & 3 \\
$D$ & 12 & 4 & 20 & 4 \\
$E$ & 25 & 8 & 37 & 5 \\
$F$ & 38 & 8 & 50 & 10 \\
\hline
\end{tabular}


Table 11. Values of our parameters used in the model.

\begin{tabular}{lcl}
\hline Parameter & Value & \multicolumn{1}{c}{ Description } \\
\hline$\alpha_{1}$ & $\frac{1}{5}$ & The coefficient of the first performance measure in the objective function \\
$\alpha_{2}$ & $\frac{1}{5}$ & The coefficient of the second performance measure in the objective function \\
$\alpha_{3}$ & $\frac{1}{5}$ & The coefficient of the third performance measure in the objective function \\
$\alpha_{4}$ & $\frac{1}{5}$ & The coefficient of the fourth performance measure in the objective function \\
$\alpha_{5}$ & $\frac{1}{5}$ & The coefficient of the fifth performance measure in the objective function \\
$f_{v}$ & {$[0.68,0.80]$} & The conversion coefficient of distance traveled to travel time \\
$\delta_{i j}^{\text {min }}$ & {$[0,70]$} & The minimal time distance between service start times at every two interdependent \\
& $\delta_{i j}^{\text {min }}+[5,50]$ & The maximal time distance between service start times at every two interdependent \\
$\delta_{i j}^{\text {max }}$ & & nodes $i$ and $j$ with precedent relation (in minutes) \\
$d u r_{i}$ & {$[5,40]$} & The time duration of performing the service required by patient-related node $i$ \\
$T$ & 600,1000 & Time horizon (in minutes) \\
\hline
\end{tabular}

Table 12. The results of solving the model with two types of modeling.

\begin{tabular}{|c|c|c|c|c|c|}
\hline Size $\left(\left|P^{\mathbf{0}}\right| \times\left|P^{\mathbf{0}}\right|-\mathbf{1} \times|V|\right)$ & Instance & $\begin{array}{c}\text { Type of } \\
\text { modeling }\end{array}$ & $\boldsymbol{W}$ & $\begin{array}{l}\text { CPU } \\
\text { time }\end{array}$ & $\begin{array}{l}\text { Gap } \\
(\%)\end{array}$ \\
\hline \multirow{10}{*}{$13 \times 12 \times 3$} & \multirow{2}{*}{ B1 } & Model 1 & 180.579 & 35.45 & \multirow{2}{*}{11.185} \\
\hline & & Model 2 & 200.78 & 31.73 & \\
\hline & \multirow{2}{*}{ B2 } & Model 1 & 246.181 & 26.78 & \multirow{2}{*}{-} \\
\hline & & Model 2 & Infeasible & - & \\
\hline & \multirow{2}{*}{ B3 } & Model 1 & 302.384 & 18.22 & \multirow{2}{*}{11.021} \\
\hline & & Model 2 & 335.710 & 13.92 & \\
\hline & \multirow{2}{*}{ B4 } & Model 1 & 91.904 & 10.52 & \multirow{2}{*}{-} \\
\hline & & Model 2 & Infeasible & - & \\
\hline & \multirow{2}{*}{ B5 } & Model 1 & 207.912 & 22.44 & \multirow{2}{*}{8.848} \\
\hline & & Model 2 & 226.308 & 18.42 & \\
\hline \multirow{10}{*}{$17 \times 16 \times 3$} & \multirow{2}{*}{$\mathrm{C} 1$} & Model 1 & 493.770 & 579.97 & \multirow{2}{*}{38.727} \\
\hline & & Model 2 & 685.002 & 588.11 & \\
\hline & \multirow{2}{*}{$\mathrm{C} 2$} & Model 1 & 620.683 & 602.30 & \multirow{2}{*}{7.415} \\
\hline & & Model 2 & 666.707 & 525.53 & \\
\hline & \multirow{2}{*}{ C3 } & Model 1 & 512.577 & 638.81 & \multirow{2}{*}{ - } \\
\hline & & Model 2 & Infeasible & - & \\
\hline & \multirow{2}{*}{$\mathrm{C} 4$} & Model 1 & 351.800 & 3560.06 & \multirow{2}{*}{30.689} \\
\hline & & Model 2 & 459.764 & 3662.20 & \\
\hline & \multirow{2}{*}{ C5 } & Model 1 & 504.177 & 487.34 & \multirow{2}{*}{28.865} \\
\hline & & Model 2 & 649.708 & 395.04 & \\
\hline
\end{tabular}

As it is shown in Table 12, despite the enlargement of the problem size and a slight increase in CPU time, there would be better values of the objective function for each instance in the first type of modeling. The relative gap between them ranges from 7 to $38 \%$, which increases for larger instances. In the second type of modeling, each staff member is prevented from performing both interdependent services. This means that the return of a staff member to an already visited patient would violate the start time of the earlier visit 
Table 13. Research domain and the levels of factors in the Genetic Algorithm (GA).

\begin{tabular}{|c|c|c|c|c|c|c|}
\hline \multirow[b]{3}{*}{ Levels } & \multicolumn{6}{|c|}{ Factors } \\
\hline & \multicolumn{2}{|c|}{ Population size } & \multicolumn{2}{|c|}{ Number of generations } & \multirow[b]{2}{*}{$\begin{array}{c}\text { Crossover } \\
\text { rate }\end{array}$} & \multirow[b]{2}{*}{$\begin{array}{c}\text { Mutation } \\
\text { rate }\end{array}$} \\
\hline & $\begin{array}{c}\text { Small } \\
\text { instances }\end{array}$ & $\begin{array}{c}\text { Large } \\
\text { instances }\end{array}$ & $\begin{array}{c}\text { Small } \\
\text { instances }\end{array}$ & $\begin{array}{c}\text { Large } \\
\text { instances }\end{array}$ & & \\
\hline 1 & 100 & 200 & 70 & 100 & 0.7 & 0.1 \\
\hline 2 & 150 & 230 & 100 & 150 & 0.8 & 0.2 \\
\hline 3 & 200 & 270 & 150 & 200 & 0.9 & 0.3 \\
\hline
\end{tabular}

and create a cycle in the route of the staff member. In other words, the same staff member cannot perform the second interdependent service for one single patient.

It should also be noted that contrary to the first model, there would be an infeasible solution in the second model for the situation where only one staff member is available to provide both interdependent services for a patient. The main reason for this issue is that the structure of the second model is designed in such a way that no sub-cycle in the final routes could be created. As obvious in Table 12, the first model ends up with feasible solutions in all situations while its objectives are more suitable compared to those in the second model.

\subsubsection{The performance of the $G A$}

As mentioned earlier, due to the complexity of our proposed model, if the problem scale is large enough, it is likely that the best solution cannot be found within a reasonable time using the current accurate methods. Therefore, we extended a GA to cope with such problems. We demonstrate how this algorithm could end up with high-qualified solutions (near-optimal solutions) in some small- and medium-sized test instances. Then, we report the final solutions obtained using this algorithm for some large-scale problems where the current exact methods are usually able to find any solutions. It should be noted that all the results of GAMS are extracted within 60000 seconds of CPU time.

\subsubsection{Parameter setting and taguchi design}

As we have some kind of generating random numbers or sequences when using metaheuristic algorithms, different final routes could be obtained at the end of all implementations. A metaheuristic algorithm for solving an optimization model is a reliable solution scheme if the final solutions in all implementations are meaningfully close to each other. To have such a solution quality, the respective parameters including population size, number of generations, crossover probability, and mutation probability should be properly adjusted. These parameters need to be optimally set through running a variety of experiments in differ- ent problem instances. We must employ a suitable technique in the design of experiments to find the optimal level of efficient parameters. We used the Taguchi method, a well-known approach for adjusting the parameters of the metaheuristic algorithms. This approach can remarkably reduce the number of experiments for parameter tuning Fraley et al. [40]. Here, we used Minitab 16 to implement Taguchi designs and parameter tuning. The research domain and the respective levels of factors are recorded in Table 13.

Based on the suggestion of the Minitab, we selected the nine-experiment option. The solution scheme is then implemented multiple times using the respective levels in each experiment for both small and large instances. The best solution amongst all the implementations for each experiment is selected as the final solution. Signal to noise $\left(\frac{S}{N}\right)$, as the first priority, is formulated as equation $\eta=\left(\frac{S}{N}\right)=$ $-10 \log \left(\frac{1}{m}\right) \sum_{i}\left(y_{i}{ }^{2}\right)$, where $m$ is the number of experiments and $y_{i}$ is the response of the process in the $i$ th experiment. The average value of the objective function and the average value of CPU time are also chosen as the second and third priorities, respectively. Therefore, the higher $\frac{S}{N}$ ratio with the lower average of the objective function and the CPU time is more suitable for decision-maker/s and the respective level of parameters should be selected. The optimal and suitable values for the GA parameters with consideration of priorities are demonstrated in Table 14.

\subsubsection{Results analysis}

In this section, the results obtained through GAMS and GA are compared with each other. It is worth mentioning that GAMS uses the Branch and Bound (B\&B) algorithm to solve optimization models. The following table demonstrates the LB obtained through GAMS, the value of the objective functions obtained using GAMS $\left(W_{B \& B}\right)$ and the GA-based solution scheme $\left(W_{G A}\right)$, and the CPU times (in seconds) for all generated problems. In addition, the relative gap between $L B$ and $W_{B \& B}$, reported by GAMS, is demonstrated in the fifth column, which is calculated using the equation relative gap $=\frac{\left(W_{B \& B}-L B\right)}{W_{B \& B}}$. The 
Table 14. Optimal level of controllable factors in the Genetic Algorithm (GA).

\begin{tabular}{lcc}
\hline Parameters & $\begin{array}{c}\text { The optimal level } \\
\text { for small problems }\end{array}$ & $\begin{array}{c}\text { The optimal level } \\
\text { for large problems }\end{array}$ \\
\hline Population size & 100 & 230 \\
Number of generations & 100 & 200 \\
Crossover probability & 0.7 & 0.9 \\
Mutation probability & 0.1 & 0.3 \\
\hline
\end{tabular}

Table 15. Numerical results for test instances.

\begin{tabular}{|c|c|c|c|c|c|c|c|c|}
\hline \multirow[b]{2}{*}{$\operatorname{Size}\left(\left|P^{0}\right| \times\left|P^{0}\right|-1 \times|V|\right)$} & \multirow[b]{2}{*}{ Instance } & \multirow[b]{2}{*}{ LB } & \multicolumn{3}{|c|}{ B\&B algorithm } & \multicolumn{2}{|c|}{ GA } & \multirow[b]{2}{*}{ Gap (\%) } \\
\hline & & & $W_{B \& B}$ & $\begin{array}{l}\text { relative } \\
\text { gap (\%) }\end{array}$ & $\begin{array}{l}\text { CPU } \\
\text { time }\end{array}$ & $W_{G A}$ & $\begin{array}{l}\text { CPU } \\
\text { time }\end{array}$ & \\
\hline \multirow{5}{*}{$8 \times 7 \times 2$} & $\mathrm{~A} 1$ & 45.959 & 45.959 & 0.00 & 0.76 & 45.959 & 29.19 & 0.00 \\
\hline & $\mathrm{A} 2$ & 72.552 & 72.552 & 0.00 & 0.38 & 72.552 & 33.56 & 0.00 \\
\hline & A3 & 46.247 & 46.247 & 0.00 & 0.25 & 46.256 & 28.99 & 0.02 \\
\hline & $\mathrm{A} 4$ & 75.054 & 75.054 & 0.00 & 0.36 & 75.064 & 28.77 & 0.01 \\
\hline & A5 & 39.561 & 39.561 & 0.00 & 0.34 & 39.561 & 28.24 & 0.00 \\
\hline \multirow{5}{*}{$13 \times 12 \times 3$} & B1 & 180.579 & 180.579 & 0.00 & 35.45 & 182.843 & 77.99 & 1.25 \\
\hline & $\mathrm{B} 2$ & 246.181 & 246.181 & 0.00 & 26.78 & 247.195 & 76.62 & 0.41 \\
\hline & B3 & 302.384 & 302.384 & 0.00 & 18.22 & 304.685 & 85.47 & 0.76 \\
\hline & B4 & 91.904 & 91.904 & 0.00 & 10.52 & 93.116 & 83.20 & 1.32 \\
\hline & B5 & 207.912 & 207.912 & 0.00 & 22.44 & 210.592 & 69.54 & 1.29 \\
\hline \multirow{5}{*}{$17 \times 16 \times 3$} & $\mathrm{C} 1$ & 493.777 & 493.777 & 0.00 & 579.97 & 508.672 & 154.66 & 3.02 \\
\hline & $\mathrm{C} 2$ & 620.683 & 620.683 & 0.00 & 602.30 & 633.813 & 140.59 & 2.12 \\
\hline & $\mathrm{C} 3$ & 512.577 & 512.577 & 0.00 & 638.81 & 527.036 & 195.65 & 2.82 \\
\hline & $\mathrm{C} 4$ & 351.800 & 351.800 & 0.00 & 3560.06 & 357.279 & 184.45 & 1.56 \\
\hline & $\mathrm{C} 5$ & 504.177 & 504.177 & 0.00 & 487.34 & 515.635 & 143.73 & 2.27 \\
\hline \multirow{5}{*}{$20 \times 19 \times 4$} & D1 & 155.788 & 817.439 & 80.94 & 60000 & 815.006 & 1380.40 & -0.30 \\
\hline & D2 & 146.228 & 911.223 & 83.95 & 60000 & 903.769 & 1277.31 & -0.82 \\
\hline & D3 & 401.991 & 708.942 & 43.30 & 60000 & 709.452 & 1224.91 & 0.07 \\
\hline & $\mathrm{D} 4$ & 660.352 & 660.352 & 0.00 & 40827.02 & 664.837 & 1379.746 & 0.68 \\
\hline & D5 & 236.692 & 742.703 & 68.13 & 60000 & 730.706 & 1236.88 & -1.62 \\
\hline \multirow{5}{*}{$37 \times 36 \times 5$} & E1 & - & - & - & 60000 & 1346.160 & 5105.85 & - \\
\hline & $\mathrm{E} 2$ & - & - & - & 60000 & 1395.275 & 4949.08 & - \\
\hline & E3 & - & - & - & 60000 & 1620.380 & 4932.52 & - \\
\hline & $\mathrm{E} 4$ & - & - & - & 60000 & 1527.837 & 4889.09 & - \\
\hline & E5 & - & - & - & 60000 & 1385.675 & 4834.36 & - \\
\hline \multirow{5}{*}{$50 \times 49 \times 10$} & $\mathrm{~F} 1$ & - & - & - & 60000 & 1640.960 & 10847.36 & - \\
\hline & $\mathrm{F} 2$ & - & - & - & 60000 & 1762.986 & 10601.17 & - \\
\hline & F3 & - & - & - & 60000 & 1926.015 & 10134.23 & - \\
\hline & $\mathrm{F} 4$ & - & - & - & 60000 & 1903.256 & 10133.94 & - \\
\hline & F5 & - & - & - & 60000 & 2190.284 & 10290.16 & - \\
\hline
\end{tabular}

last column represents the gap between $W_{B \& B}$ and $W_{G A}$ computed using the following equation:

$$
g a p=\left(\frac{W_{G A}-W_{B \& B}}{W_{B \& B}}\right) \times 100 .
$$

As it is illustrated in Table 15, the optimal values of the objective function are almost the same as the objective function values achieved using the GA in small- and medium-sized instances (in sets A, B, and C) and the gap is only at most $3.02 \%$. Another interesting point is that all instances in sets $\mathrm{A}, \mathrm{B}$, and $\mathrm{C}$ are optimized within at most 638 seconds using B\&B except C4, while GA ends up with almost the same objectives in at most 195.65 seconds, which are reasonably less than the CPU times observed by 
B\&B. The large-sized instances in set $\mathrm{D}$ cannot be solved optimally in 60,000 seconds using B\&B and only feasible solutions are achieved. However, GA could obtain feasible solutions with close objective values to those obtained through GAMS in quite shorter CPU times (at most 1380.40 seconds). The relative gaps for these instances fluctuate between $43 \%$ and $84 \%$, except for D4 that is solved exactly within 40827.02 seconds (i.e., the relative gap is zero). The gaps are $-0.30 \%,-0.82 \%, 0.07 \%$, and $-1.62 \%$ for D1, D2, D3, and D5, respectively. A negative gap means that the objective function value of $\mathrm{GA}$ is better than that of B\&B (it should also be noted that GAMS cannot solve the optimality of these instances). However, GA saves at least $98 \%$ of the CPU time considered by $\mathrm{B} \& \mathrm{~B}$ to obtain the same objective function value in order to obtain the same objective function value for all instances in set $\mathrm{D}$. Therefore, a significant improvement can be obtained in less computational time. For large instances in sets $\mathrm{E}$ and $\mathrm{F}$, the B\&B algorithm could not find any feasible solution and any LBs within 60000 seconds; however, GA finds feasible solutions within maximum 10847.36 seconds (about $3 \mathrm{~h}$ ), which is certainly a practical time for making decisions. These results confirm that the solution scheme could suitably obtain optimal or near-optimal solutions within a limited computational time even for large-sized instances.

Furthermore, Figure 16 also illustrates that the objectives for both exact and metaheuristic methods are almost the same for small- and medium-sized instances. However, as the size of the problems increases, exact methods cannot yield a solution within a reasonable time while the GA leads to a feasible solution in a more limited time. In Figures 17 and 18 the trend of convergence of GA for instances D3 and F1 are depicted.

\section{Conclusions}

The primary goal of paying much attention to home health care is to improve the welfare of communities by providing high-quality services to satisfy people's health requirements in their homes. The home health care problem involves staff members routing and services scheduling in which the performance measures such as total system costs and patient/staff satisfaction levels are optimized. In this regard, we developed a comprehensive mathematical model in which all staff members could have specific qualifications and skills (heterogeneous staff members); therefore, they can provide some limited services compatible with their defined abilities. Whereas each staff member could have his/her own vehicle, she/he can traverse a distance between two nodes less than others, that is, the speed of vehicles could be different. The interesting feature of

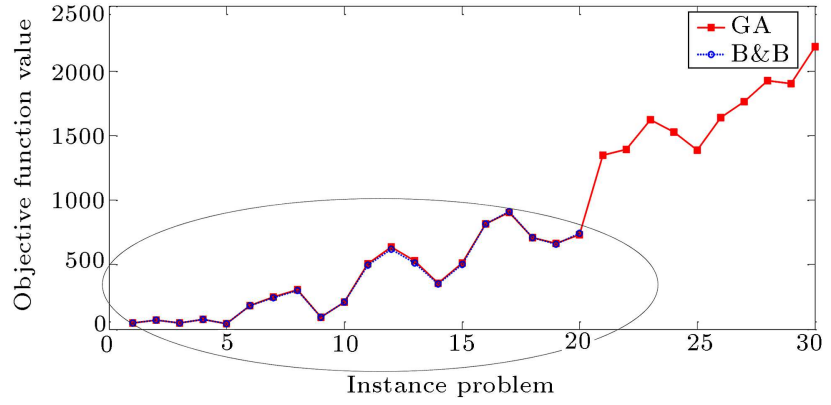

Figure 16. Comparison of the value of the objective function using B\&B and Genetic Algorithm (GA).

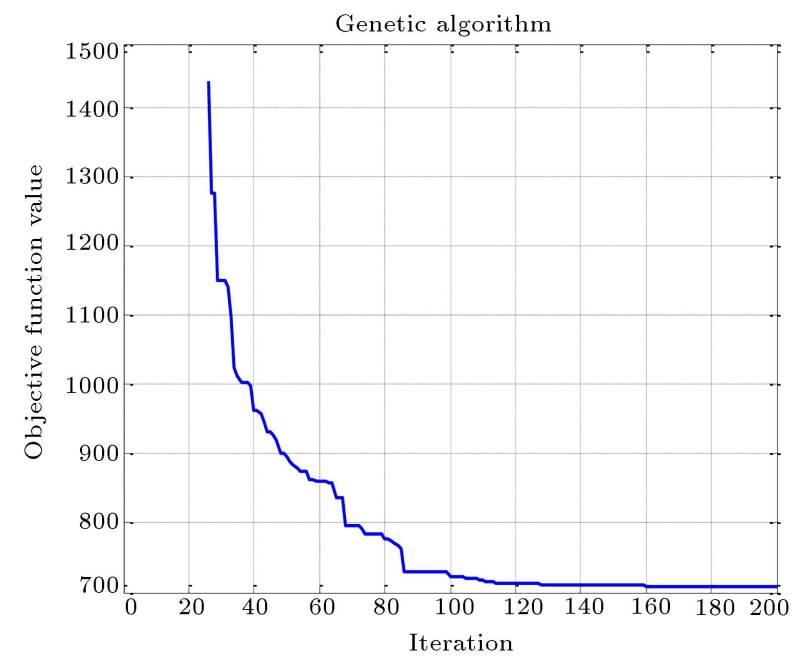

Figure 17. Convergence diagram of the example D3.

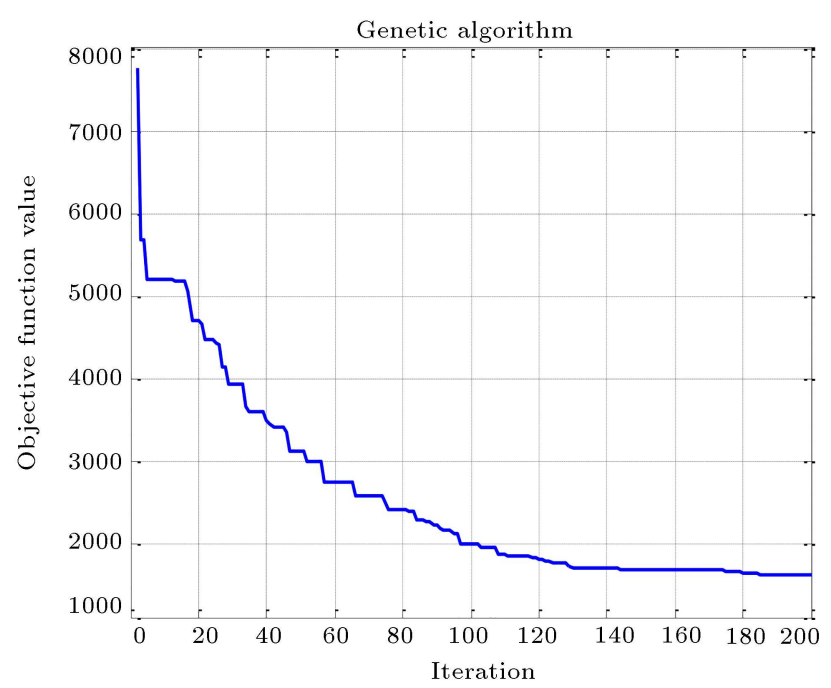

Figure 18. Convergence diagram of the example F1.

the proposed model, which would make it more useful in real situations, is that the staff members could work over the working times approved in their own contracts (i.e., the consideration of overtime in the model). It is also assumed that all service requirements should be provided within predefined time windows for both 
patients and staff members. To make the model more flexible, the starting time of patients time windows is considered as a hard constraint, while its ending value is a soft constraint. In addition, all staff members could have a break period within a routine day. Compared with other works in the previous literature, the remarkable feature of the model we developed is that it also considers Continuity of Care ( $\mathrm{CoC})$, related required services, and blood sampling requirements. In other words, proper preferences in choosing desired staff members could be done by patients, the respective services could be accomplished simultaneously or with predefined precedence, and all blood samples must be returned to the blood center (i.e., central depot) within a specified time window.

To incorporate all these features into our mathematical model, we constructed the respective home health care network using some virtual nodes. The interesting point is that due to the nature of the model and its respective modification, the model ends up with a feasible solution in all different situations. This means that all required services are eventually responded by the qualified staff members; however, they could be implemented with some delay. Moreover, we used our resources in an effective way so that one staff member can provide two interdependent services with precedent relations. Therefore, the total cost could be remarkably decreased and sufficient time/money could be saved. In addition, since the $\mathrm{CoC}$ is incorporated into the proposed model, both patients and staff members would have more friendly relations which leads to higher patients satisfaction. There is also no limitation in our proposed model to apply it in daily, weekly, or even monthly Home Healthcare Routing and Scheduling Problem (HHCRSP). Furthermore, there is no serious restriction to consider different blood, break, and depot centers for each staff member. The proposed optimization model could be simply customized to new problems with multiple nodes for these centers.

Due to the complexity of the model, it cannot be solved through traditional optimization methods for real-life problems. Therefore, a Genetic Algorithm (GA) has been extended which can be used for most routing and scheduling problems. By comparing the developed algorithm with the exact solutions of small and medium-scale problems, the efficiency of our proposed solution could be revealed.

The model can be extended for a stochastic situation where some respective parameters are uncertain. A dynamic approach could also be presented in which the process of accepting (canceling) the new (existing) patients and rescheduling the required services are taken into account. Moreover, two different situations, which include performing a certain visit and transporting medical facilities and medicine between the health care institutions and the patients homes, can also be incorporated into the model.

\section{References}

1. Tarricone, R. and Tsouros, A.D., Home Care in Europe: the Solid Facts, WHO Regional Office Europe (2008).

2. Bouajaja, S. and Dridi, N. "A survey on human resource allocation problem and its applications", $O p$ erational Research, 17(2), pp. 339-369 (2017).

3. Pouraliakbarimamaghani, M., Mohammadi, M., and Mirzazadeh, A. "A queuing location-allocation model for a capacitated health care system", Scientia Iranica, E., 24, pp. 751-764 (2017).

4. Bredström, D. and Rönnqvist, M. "Combined vehicle routing and scheduling with temporal precedence and synchronization constraints", European Journal of $O p$ erational Research, 191(1), pp. 19-31 (2008).

5. Kergosien, Y., Lenté, C., and Billaut, J.-C. "Home health care problem: An extended multiple traveling salesman problem", Proc., 4th Multidisciplinary International Conference on Scheduling: Theory and Applications (MISTA'09), Dublin (Irlande), pp. 10-12 (2009).

6. Rasmussen, M.S., Justesen, T., Dohn, A., and Larsen, J. "The home care crew scheduling problem: Preference-based visit clustering and temporal dependencies", European Journal of Operational Research, 219(3), pp. 598-610 (2012).

7. Mankowska, D.S., Meisel, F., and Bierwirth, C. "The home health care routing and scheduling problem with interdependent services", Health Care Management Science, 17(1), pp. 15-30 (2014).

8. Lanzarone, E. and Matta, A. "Robust nurse-to-patient assignment in home care services to minimize overtimes under continuity of care", Operations Research for Health Care, 3(2), pp. $48-58$ (2014).

9. Elbenani, B., Ferland, J.A., and Gascon, V. "Scatter search approach for solving a home care nurses routing and scheduling problem", In University of Montreal, Canada (2011).

10. Wirnitzer, J., Heckmann, I., Meyer, A., and Nickel, S. "Patient-based nurse rostering in home care", Operations Research for Health Care, 8, pp. 91-102 (2016).

11. Laporte, G. "The vehicle routing problem: An overview of exact and approximate algorithms", European Journal of Operational Research, 59(3), pp. 345358 (1992).

12. Toth, P. and Vigo, D., Vehicle Routing: Problems, Methods, and Applications, Siam (2014).

13. Eksioglu, B., Vural, A.V., and Reisman, A. "The vehicle routing problem: A taxonomic review", Computers \& Industrial Engineering, 57(4), pp. 1472-1483 (2009). 
14. Gutiérrez, E.V. and Vidal, C.J. "Home health care logistics management: Framework and research perspectives", International Journal of Industrial Engineering and Management, 4(3), pp. 173-182 (2013).

15. Fikar, C. and Hirsch, P. "Home health care routing and scheduling: A review", Computers \& Operations Research, 77, pp. 86-95 (2017).

16. Begur, S.V., Miller, D.M., and Weaver, J.R. "An integrated spatial DSS for scheduling and routing home-health-care nurses", Interfaces, 27(4), pp. 35-48 (1997).

17. Cheng, E. and Rich, J.L. "A home health care routing and scheduling problem", URL http://citeseerx. ist. psu. edu/viewdoc/summary (1998).

18. Bertels, S. and Fahle, T. "A hybrid setup for a hybrid scenario: combining heuristics for the home health care problem", Computers \& Operations Research, 33(10), pp. 2866-2890 (2006).

19. Eveborn, P., Flisberg, P., and Rönnqvist, M. "Laps care an operational system for staff planning of home care", European Journal of Operational Research, 171(3), pp. 962-976 (2006).

20. Bräysy, O., Dullaert, W., and Nakari, P., Municipal Routing Problems: A Challenge for Researchers and Policy Makers? In: Witlox FJA, Ruijrok CJ (eds) Bijdragen Vervoerslogistieke Werkdagen, Nautilus Academic Books, Zelzate, pp 330-347 (2007).

21. Akjiratikarl, C., Yenradee, P., and Drake, P.R. "PSObased algorithm for home care worker scheduling in the UK", Computers \& Industrial Engineering, 53(4), pp. 559-583 (2007).

22. Trautsamwieser, A. and Hirsch, P. "Optimization of daily scheduling for home health care services", Journal of Applied Operational Research, 3(3), pp. 124-136 (2011).

23. Hiermann, G., Prandtstetter, M., Rendl, A., Puchinger, J., and Raidl, G.R. "Metaheuristics for solving a multimodal home-healthcare scheduling problem", Central European Journal of Operations Research, 23(1), pp. 89-113 (2015).

24. Duque, P.M., Castro, M., Sörensen, K., and Goos, P. "Home care service planning. The case of landelijke thuiszorg", European Journal of Operational Research, 243(1), pp. 292-301 (2015).

25. Redjem, R. and Marcon, E. "Operations management in the home care services: a heuristic for the caregivers, routing problem", Flexible Services and Manufacturing Journal, 28(1-2), pp. 280-303 (2016).

26. Frifita, S., Masmoudi, M., and Euchi, J. "General variable neighborhood search for home healthcare routing and scheduling problem with time windows and synchronized visits", Electronic Notes in Discrete Mathematics, 58, pp. 63-70 (2017).
27. Decerle, J., Grunder, O., El Hassani, A.H., and Barakat, O. "A memetic algorithm for a home health care routing and scheduling problem", Operations Research for Health Care, 16, pp. 59-71 (2018).

28. Liu, R., Yuan, B., and Jiang, Z. "A branch-andprice algorithm for the home-caregiver scheduling and routing problem with stochastic travel and service times", Flexible Services and Manufacturing Journal, 31(4), pp. 989-1011 (2019).

29. Demirbilek, M., Branke, J., and Strauss, A. "Dynamically accepting and scheduling patients for home healthcare", Health Care Management Science, 22(1), pp. 140-155 (2019).

30. Regis-Hernández, F., Carello, G., and Lanzarone, E. "An optimization tool to dimension innovative home health care services with devices and disposable materials", Flexible Services and Manufacturing Journal, 32(3), pp. 1-38 (2020).

31. Baker, B.M. and Ayechew, M. "A genetic algorithm for the vehicle routing problem", Computers \& Operations Research, 30(5), pp. 787-800 (2003).

32. Liu, S., Huang, W., and Ma, H. "An effective genetic algorithm for the fleet size and mix vehicle routing problems", Transportation Research Part E: Logistics and Transportation Review, 45(3), pp. 434-445 (2009).

33. Ghoseiri, K. and Ghannadpour, S.F. "Multi-objective vehicle routing problem with time windows using goal programming and genetic algorithm", Applied Soft Computing, 10(4), pp. 1096-1107 (2010).

34. Tasan, A.S. and Gen, M. "A genetic algorithm based approach to vehicle routing problem with simultaneous pick-up and deliveries", Computers \& Industrial Engineering, 62(3), pp. 755-761 (2012).

35. Wang, H.-F. and Chen, Y.-Y. "A genetic algorithm for the simultaneous delivery and pickup problems with time window", Computers \& Industrial Engineering, 62(1), pp. 84-95 (2012).

36. Liu, R., Xie, X., Augusto, V., and Rodriguez, C. "Heuristic algorithms for a vehicle routing problem with simultaneous delivery and pickup and time windows in home health care", European Journal of Operational Research, 230(3), pp. 475-486 (2013).

37. Pankratz, G. "A grouping genetic algorithm for the pickup and delivery problem with time windows", Or Spectrum, 27(1), pp. 21-41 (2005).

38. Holland, J.H., Adaptation in Natural and Artificial Systems: an Introductory Analysis with Applications to Biology, Control, and Artificial Intelligence, U Michigan Press (1975).

39. Gen, M., Cheng, R., and Lin, L., Network Models and Optimization: Multiobjective Genetic Algorithm Approach, Springer Science \& Business Media (2008). 
40. Fraley, S., Oom, M., Terrien, B., and Date, J. "Design of experiments via Taguchi methods: orthogonal arrays", The Michigan Chemical Process Dynamic and Controls Open Text Book, USA, 2(3), p. 4 (2006).

\section{Biographies}

Zahra Entezari received a BSc degree in applied mathematics from Alzahra University in 2013 and a MSc degree in Industrial Engineering from Amirkabir University of Technology in 2016. Now, she is a PhD candidate in Industrial Engineering and Management Systems Department at the Amirkabir University of Technology, Tehran, Iran. Her research interests are stochastic programming, simulation-based optimiza- tion and also, machine learning methods in health systems.

Masoud Mahootchi received BSc and MSc degrees in Industrial Engineering Department from Amirkabir University of Technology in 1993 and 1997, respectively. He also received his $\mathrm{PhD}$ in Systems Design Engineering from the University of Waterloo (2009) and he is now a faculty member at the Amirkabir University, Tehran, Iran. He focused on storage management under uncertainty using nonlinear and soft computing methods during his $\mathrm{PhD}$ at present, he is teaching the courses of simulation-based optimization, Portfolio optimization, and health care optimization methods at the Amirkabir University. 